\title{
Design and Characterization of Programmable DNA Nanotubes Supporting Information
}

\author{
Paul W. K. Rothemund, ${ }^{*}, \dagger$ Axel Ekani-Nkodo, ${ }^{\ddagger}, \S$ Nick Papadakis, ${ }^{\dagger}$ \\ Ashish Kumar, ${ }^{\ddagger}$ Deborah Kuchnir Fygenson, $, ₫, \S$ Erik Winfree ${ }^{*, \dagger}$ \\ Contribution from the Departments of Computer Science and Computation and Neural Systems, \\ California Institute of Technology, Pasadena, CA 91125 and the Department of Physics, \\ University of California Santa Barbara, Santa Barbara, CA 93106 \\ E-mail:pwkr@dna.caltech.edu
}

\section{Methods}

Design, data-processing and modelling: DNA sequences were designed using our own algorithms based on sequence symmetry minimization implemented in Matlab and $\mathrm{C}$ (available at http://www.dna.caltech.edu/DNAdesign/). Curve fits for persistence length data and models for lattice strain energies were calculated in Matlab. Molecular models were constructed and visualized using a combination of NAMOT, RasMol, and PyMol (scripts and coordinates at http://www.dna.caltech.edu/SupplementaryMaterial/). The molecular models are for visualization only and have not been subjected to molecular dynamics calculations.

DNA sample preparation: Lyophilized HPLC- or PAGEpurified DNA oligonucleotides were purchased from Integrated DNA Technologies (Coralville, IA), resuspended in water, quantitated by UV absorbance at $260 \mathrm{~nm}$, and stored at $-20^{\circ} \mathrm{C}$. All samples were prepared in a $1 \mathrm{X}$ Tris-Acetate-EDTA (TAE) buffer with $12.5 \mathrm{mM}$ magnesium acetate $(\mathrm{pH}=8.3)$. An equimolar mixture of strands (5 strands if one tile, 10 strands if two) was annealed from $95^{\circ} \mathrm{C}$ to $25^{\circ} \mathrm{C}$ at $1^{\circ} \mathrm{C} /$ minute in $0.1^{\circ} \mathrm{C}\left(\mathrm{AFM}\right.$ ) or $5^{\circ} \mathrm{C}$ (fluorescence microscopy) steps in a PCR machine (Eppendorf Mastercycler). For AFM, each strand was present at $200 \mathrm{nM}$, for fluorescence microscopy the total concentration of tiles was kept at $400 \mathrm{nM}$. For fluorescence microscopy, a single fluorescein-labeled strand was incorporated into each tile; the position of the dye was varied from the $5^{\prime}$ end of the \#3 strand to the $5^{\prime}$ end of the \#5 strand with no apparent effect. AFM of REp+SEp(3:FAM) was similar to that of REp+SEp.

Preparation of PVP coated glass: Adapted from. ${ }^{1}$ Microscope slides and coverslips were washed in $1 \mathrm{M} \mathrm{NaOH}$ for 1 hour, rinsed thoroughly with de-ionized (DI) water and immersed in $1 \% \mathrm{v} / \mathrm{v}$ acetic acid solution for 2 hours. Then, they were rinsed again with DI water and silanized in a $1 \% \mathrm{v} / \mathrm{v} 3-$ (trimethoxysilyl)propylmethacrylate (Aldrich) in $1 \% \mathrm{v} / \mathrm{v}$ acetic acid for 36 hours. For polymer coating, $500 \mathrm{~mL}$ of a $4 \% \mathrm{w} / \mathrm{v} M_{w}=$ 360,000 poly(vinylpyrrolidone) (PVP, USB Corp.) solution with $2.5 \mathrm{~mL}$ of $10 \% \mathrm{w} / \mathrm{w}$ ammonium persulfate solution and $250 \mu \mathrm{l}$ of $\mathrm{N}, \mathrm{N}, \mathrm{N}^{\prime}, \mathrm{N}^{\prime}$-tetramethylethylenediamine (TEMED, Acros) was prepared. Slides and coverslips were incubated in the PVP solution at $80^{\circ} \mathrm{C}$ for 18 hours. They were then rinsed and stored in DI water. Coating was stable for at least 2 weeks.

Preparation for fluorescence microscopy: Samples were left

\footnotetext{
${ }^{*}$ Department of Computer Science, California Institute of Technology

${ }^{\dagger}$ Computation and Neural Systems, California Institute of Technology

${ }^{\ddagger}$ Physics Department, University of California, Santa Barbara

${ }^{\S}$ Materials Research Laboratory, University of California, Santa Barbara

๑ Biomolecular Science and Engineering Program, University of California, Santa Barbara
}

overnight at room temperature after annealing. Immediately prior to use, a PVP-coated microscope slide and coverslip were rinsed with ethanol and dried. Then, $2.6 \mu \mathrm{l}$ of solution containing DNA tubes and oxygen scavenging system $(0.035 \mathrm{mg} / \mathrm{ml}$ catalase, $0.2 \mathrm{mg} / \mathrm{ml}$ glucose oxidase, $4.5 \mathrm{mg} / \mathrm{ml}$ glucose, $5 \% \quad \beta$-mercaptoethanol) was deposited onto the slide, covered with the coverslip and sealed with epoxy or parafin. The distance between slide and coverslip was $\approx 5 \mu \mathrm{m}$ and the thickness of sample solution was typically narrowed to $\approx 3 \mu \mathrm{m}$ by the PVP coating.

Fluorescence microscopy: Samples were imaged on an inverted microscope (IX 70, Olympus) with 100X/1.40 NA oil immersion and 40X/0.75 NA air objectives. Blue light was filtered from a mercury arc lamp through an interference filter $(475 \pm 40 \mathrm{~nm})$ for excitation. The emitted fluorescence passed through a dichroic mirror (505 DRLP) and a green interference filter $(525 \pm 45 \mathrm{~nm})$. Images were recorded to a computer RAM via a cooled CCD camera (Sensicam, Cooke) using the corresponding software (CamWare, Cooke). Images were processed in ImageJ (Java version of NIH Image: http://rsb.info.nih.gov/ij/) by performing the following operations: invert, binarize, open (erosion followed by dilation to smooth and remove isolated pixels) and close (dilation followed by erosion to smooth and fill small holes). Finally, images were skeletonized to obtain contours 1 pixel wide. Contour lengths were measured using the Analyze Particles macro in ImageJ. To guarantee that tubes that were measured were essentially confined to $2 \mathrm{D}$, only those tubes whose whole contour remained in the $\approx 1 \mu \mathrm{m}$ focus were scored.

Atomic force microscopy: AFM imaging was performed in Tapping Mode under TAE/ $\mathrm{Mg}^{2+}$ buffer on a Digital Instruments Nanoscope III (Veeco) equipped with a nanoAnalytics Q-control III (Asylum Research) and a vertical engage J-scanner, using the $\approx 9.4$ $\mathrm{kHz}$ resonance of the narrow $100 \mu \mathrm{M}, 0.38 \mathrm{~N} / \mathrm{m}$ force constant cantilever of an NP-S oxide-sharpened silicon nitride tip (Veeco Metrology). After self-assembly is complete, samples were prepared for AFM imaging by deposition of $\approx 5 \mu \mathrm{l}$ onto a freshly-cleaved mica surface (Ted Pella) attached by hot melt glue to a $15 \mathrm{~mm}$ metal puck; an additional $30 \mu \mathrm{l}$ of buffer was added to both sample and cantilever (mounted in the standard Tapping Mode fluid cell) before the sample and fluid cell were positioned in the AFM head. (When observation of tubes falling events was desired, $30 \mu \mathrm{l}$ of buffer was added to the mica first, and then the $5 \mu \mathrm{l}$ of sample were added.) The tapping amplitude setpoint, after engage, was $0.2-0.4$ volts, the drive amplitude was 100-150 millivolts, and scan rates ranged from 2-5 Hz. Individual tiles are most clearly resolved at low amplitude setpoint and high drive amplitude values. Under such conditions, the greatest damage is done to the sample and the hairpin labels are 
less distinct, sometimes disappearing entirely. To prevent damage to samples, amplitude setpoint was maximized and/or drive amplitude minimized subject to the constraint that tiles and their hairpin labels be visible. After acquisition, images were flattened by subtracting a low-order polynomial from each scan line, or by adjusting each scan line to match intensity histograms. To create movies, multiple scans were aligned in Matlab using hand-picked fiducial marks as guides. The scale in AFM images was calibrated to the width of the tiles; previous experience has shown this to be a reliable standard.

Gels for probing tube structure: For each strand SE5 with a biotin-dT (with a 6 carbon linker) added $5^{\prime}$ of nucleotides 6 through $31(\mathrm{X}=6-31)$ we ran a gel testing the binding of streptavidin-Cy3 to the strand (a) alone, (b) in a SE tile without sticky ends SEn(3:FAM, $5: \mathrm{b} X),(\mathrm{c})$ in a flipped tube SEs(1:h22,3:FAM, 5:bX) and (d) normal tube SEs(3:FAM, 5:bX). RE4-DIAG was added as a marker to the strand and tile samples. For all streptavidin binding experiments a $1 \mu \mathrm{l}$ of streptavidin-Cy3 conjugate (Sigma S6402, 3-9 Cy3 per streptavidin, diluted $\approx 1: 15$ in water) or $1 \mu \mathrm{l}$ of water (controls) was added to $10 \mu \mathrm{l}$ of strands ( $1 \mu \mathrm{M}$ SE5-bX, 2 uM RE4-DIAG), tiles (.16 $\mu \mathrm{M}$ tiles, $1.6 \mu \mathrm{M}$ RE4-DIAG), flipped tubes (.2 $\mu \mathrm{M}$ tiles) or normal tubes ( $.2 \mu \mathrm{M}$ tiles), mixed by pipette, and allowed to bind 10 minutes. Then $1 \mu \mathrm{l}$ of $10 \mathrm{mM}$ biotin solution was added to quench the reaction, mixed by pipette, followed by $2 \mu \mathrm{l}$ bromophenol blue loading dye (xylene cyanol interfered with the interpretation of some bands). Gels were loaded with 5-6 $\mu$ l of solution/lane and run immediately. (Within 30 minutes; we found that if quenched samples were allowed to sit overnight, the biotin quench would displace the DNA strands from the biotin and no labelling was observed.) Strands and tiles were run in $10 \%$ non-denaturing polyacrylamide gels (19:1 bis:acrylamide, $15 \mathrm{~V} / \mathrm{cm}$ for $\approx 2$ hour for SE5 strands, $\approx 1$ hour for SE tiles, maintained at $4^{\circ} \mathrm{C}$ ) stained 20 minutes with Sybr Green I (Molecular Probes) and imaged on a Biorad Molecular Imager FX gel scanner (excited at 488, imaged with 530 bandpass filter). The biotinylated strands gel showed biotinylation efficiences from $52 \%$ to $82 \%$; it was so noisy that we did not use it to normalize other results. Flipped and normal tubes were run in .3\% agarose (Seakem gold, $8 \mathrm{~V} / \mathrm{cm}$, for 30 minutes cooled so the buffer remained $<20^{\circ} \mathrm{C}$ ) and imaged unstained (green channel: excited at $488 \mathrm{~nm}$, imaged with $530 \mathrm{~nm}$ bandpass filter; red channel: excited at 532, imaged with a $555 \mathrm{~nm}$ long-pass filter); no staining was necessary because in these gels all species of interest are labelled with SE3-FAM. The agarose gels just serve to separate tubes from free streptavidin and unbound tiles from the tubes - the tubes never left the wells; care was taken not to rinse them out during gel handling.

\section{Effects of hairpins on lattice-mica adhesion}

In general, in the presence of $\mathrm{Mg}^{2+}$, DNA tile lattices bind very strongly to mica. (Lattices bind more strongly with $\mathrm{Mg}^{2+}$ than does double-stranded DNA; even 4 tile clusters bind well enough to be routinely imaged with high resolution - double-stranded DNA rarely binds and images so well. Probably due to cooperative binding of tiles to mica, this difference is consistent with previous studies showing $\mathrm{Mg}^{2+}$ is insufficient to image double-stranded DNA. ${ }^{2}$ Our concentrations of $\mathrm{Mg}^{2+}$ are roughly 5-fold higher, however.) An exception appears to be tubes modified with hairpins on the outside: they bind well enough to be imaged, and open into sheets on the surface but we observe several phenomena that suggest they have low adhesion to mica. Closed tubes with hairpins appear to persist much longer on mica than do tubes without hairpins. Such tubes are sometimes mobile on the mica, apparently pushed around by the AFM tip: Where parallel to the scan direction such tubes appear much narrower than tubes perpendicular to the scan direction, and sometimes they disappear and reappear (often in the same spot) from scan to scan while surrounding opened tubes remain stable.

The hypothesis that tubes with hairpins adhere less to the mica surface, and are thus less prone to a mica-catalyzed tube-opening mechanism, is supported by experiments with the diagonal tubes REd+SEd(1,5:h14). Spread flat on a mica surface, a patch of REd+SEd(1,5:h14) lattice has a chirality dictated by the orientation of its hairpins: "right-handed" if the hairpins face down as in Fig. 1C and "left-handed" if the hairpins face up as in Fig. 2E. (This handedness is independent of which way the tubes actually close, however if tubes close as the model predicts then the top and bottom edges of the lattice in Fig. 1C would roll upward to form a tube with true left-handed helicity.) Pre-formed REd+SEd(1,5:h14) tubes were deposited on mica and we made two measurements of handedness: (1) that of lattices left by tubes observed to open in real-time and (2) that of lattices in the background (presumably from already open tubes). Freshly-opened lattices had a weakly biased chirality, of 23:15 (lefthanded:right-handed) but background lattices had a strong bias in favor of left-handed fragments (65:11). We observed that the stripes on freshly-opened right-handed lattice looked blurred (a possible indication of weak binding), and that these fragments sometimes flipped to become left-handed fragments (providing a mechanism for creating the bias in background lattices) or disappeared while in the same view a left-handed fragment grew. Because we know (from hairpin insertion experiments) that the hairpins are on the outside we conclude that open tubes bearing hairpins bind preferentially with their insides towards the mica; we suspect this is due to low adhesion of the hairpins but cannot exclude the possibility that it is caused by the intrinsic curvature of the lattice. This conclusion is further supported by experiments in which REd and $\operatorname{SEd}(1,5: \mathrm{h} 14)$ tiles were annealed separately and then mixed during deposition onto mica. In this case the mica surface was completely covered by small domains of left-handed fragments, that is, with hairpins pointing up.

We note that an alternative hypothesis for the effect of hairpins on tube-opening is that the hairpins themselves may stabilize tube structure and lattice curvature, perhaps by repelling each other sterically or electrostatically. Or they may prevent tip-lattice interactions that cause tubes to open.

\section{Derivation of persistence length estimate for tubes}

The persistence length of a double-helix is simply proportional to the Young's modulus, $E$, and the moment of inertia for the helix, $i$ : $p_{\text {helix }} \equiv E i / k T{ }^{3}$ Assuming that the Young's modulus for tubes is the same as for helices, the persistence length of tubes is similarly $p_{\text {tube }} \equiv E I / k T$, where $I$ is the moment of inertia for tubes. Thus $p_{\text {tube }} / p_{\text {helix }}=I / i$.

Treating a tube as a bundle of $2 N$ rigidly linked cylindrical rods of radius $r$, we can calculate $I$ in terms of $i$. Each rod, whose moment of inertia with respect to its own center of mass is $i$, is displaced from the tube's center of mass by a distance $R$ (see Fig. 1J). By the parallel axis theorem, the rod's moment of inertia with respect to the tube's center of mass is $i+M R^{2}$, where $M$ is the rod's mass. Assuming uniform density, $i=\frac{1}{2} M r^{2}$, and thus $I=2 N\left(i+M R^{2}\right)=2 N\left(i+\frac{2 i}{r^{2}} R^{2}\right)$, which immediately yields the desired result that

$$
\frac{p_{\text {tube }}}{p_{\text {helix }}}=\frac{I}{i}=2 N\left[1+2\left(\frac{R}{r}\right)^{2}\right] .
$$

\section{Notes on hairpin insertions}

Positions 8 and 29 are special ones for hairpin insertion since they position the hairpin directly over the crossover in the double 
crossover. In essence this turns the 4-arm junction that normally occurs at a crossover point into a 5-arm junction. In order for the double crossover to act as a normal tile in the context of a tube or lattice, we believe that there must be stacking across the two core helices. Addition of a hairpin at the junction (or even one might think within a base or two of the junction) may disrupt the correct stacking at this junction - the hairpin might, for example, stack against any one of the normally occurring 4 arms and leave one arm without its normal partner. Nonetheless, as can be seen in Supporting Fig. 8, the insertion of hairpins at position 8 or adjacent positions 7 or 9 does not prevent the formation of functional tiles that can assemble into normal or flipped tubes. Position 29, however, does something out of the ordinary-it forms some small lattice but it also forms, with very low abundance, what appears to be a 3-connected network of predominantly hexagons and some pentagons. This suggests that the results for position 29 do not have bearing on the question of inside $v s$ outside. That the hairpin should disrupt the crossover at position 29 and not at position 8, despite the similar sequence context around each junction for 2 bases on either side, could be due to their not being exactly equivalent positions with respect to sequence or the symmetries of the overall molecule.

For position 15, and 17 through 23, the hairpin insertion experiment was repeated with 4-base pair hairpins using tiles $\operatorname{SEs}(5: \mathrm{h} X$ short). Most of these tubes displayed flipped:normal ratios similar to that of their their 8-base pair counterparts while three $(18,19$, 22) appeared to yield lower flipped:normal ratios. In no case did shortening the hairpin completely eliminate flipped tubes at a position for which flipped tubes were observed using long hairpins and in no case did the ratio of flipped:normal increase with a decrease in hairpin length. These results are consistent with the idea that it is size-dependent steric or electrostatic effects of the hairpin (rather than the existence of a branch in the DNA backbone at a particular position) that causes the tubes to form an alternative configuration.

\section{Sticky-end truncations}

We explored the effect of stacking and sticky end length on the formation of DNA nanotubes by creating strands with single base truncations on their ends, the results of which are given in Supporting Fig. 15. Particularly interesting are cases in which bases adjacent to the sticky ends are truncated so that stacking interactions at the sticky ends are lost. For each of the 3 different single tile tubes studied (REs, SEs, and VE-00) we replaced strand \#1 or \#5 with a version truncated 1 base at its $5^{\prime}$ and 1 base on its $3^{\prime}$ end. With one such strand replaced, one half of the stacking interactions at sticky ends are removed (assuming that the unpaired base in the sticky end does not bulge out and allow stacking). For REs (5:5t1-3t1), REs(3:5t1-3t1), SEs (5:5t1-3t1), and SEs(3:5t1-3t1) we observed that only small lattice fragments (100-300 nm on a side) were formed but for VE-00(5:5t1-3t1) and VE-00(3:5t1-3t 1$)$ either entirely flipped tubes or a mixture of flipped and large normal tubes were formed. This suggests that stacking interactions are important in the geometry of double crossover lattices-in particular it suggests that despite the distortion of the helix axis implied by the $\approx 2 \mathrm{~nm}$ separation observed between tiles, the distortion is not extreme enough to destroy stacking interactions at sticky ends. It is possible that the effect of removing stacking interactions does no more than decrease the melting temperature, and that the small lattices observed for REs (5:5t1-3t1) and similar systems were nucleated and grown on the mica during sample preparation - in fact these small lattices are indistinguishable from the small lattices that we know have grown on mica in experiments where tiles REp and SEp were annealed separately and then mixed during deposition onto the mica surface for AFM imaging (data not shown). However, the result that VE-00(5:5t1-3t1) and VE-00(3:5t1-3t1) form flipped tubes in solution shows that such truncations can profoundly change the morphology of tubes. So we find it unlikely that a change in melting temperature is responsible for the lack of tubes in the other samples.

We also explored 1 base truncations of the sticky ends so that both base pair and a stacking interaction were lost. Some of these truncations, for example REs(2:EE01-5t1), result in only small lattices. Notably, one truncation, REs(4:DIAG-5t1), resulted in a mixture bearing large numbers of flipped and large normal tubes. Thus the loss of a single base pair and a stacking interaction are in this case sufficient to cause the formation of flipped tubes.

Another lesson that these studies yield is the importance of using full-length purified DNA sequences in DNA nanotechnology constructions. The greatest impurity in DNA synthesis reactions are typically $n-1$ truncation products; we have shown, however, that a single base truncation can cause a profound change in tube morphology.

\section{Arm length variations}

A question that is often asked is whether changing arm lengths of the double crossovers would change the diameter of the tubes created. The effect of changing arm lengths is to change the distance between intermolecular crossovers in the lattice that is formed. For the unmodified DAE-E tiles used here this distance, $D$, is 21 base pairs from the right crossover of one tile to the left crossover of a tile binding on its righthand side. In fact, rather than changing the natural diameter of the tubes, changing the distance $D$ serves to increase the overtwist or undertwist between crossovers and adds strain to the lattice. This is a consequence of the constraints on the major and minor groove orientations at crossovers, as shown in Fig. 1J. As $D$ is increased or decreased too much from the unstrained 2-helical turn value of 21 , we expect that tubes will no longer be able to form. We explored the ability of the tube structure to tolerate overtwist or undertwist in the helix between intermolecular crossovers by attempting to make tubes out of double crossovers with changed arm lengths. For each basic double-crossover REp and SEp we made four variants (see Supporting Fig. 3): one variant with the righthand arms increased by one basepair in length (e.g. REp-increase1 ), one variant with both the righthand and leftand arms increased by one basepair in length (e.g. REp-increase-2), one variant with the lefthand arms decreased by one basepair in length (e.g. REpdecrease-1), and one variant with both the righthand and lefthand arms decreased by one basepair in length (e.g. REp-decrease-2).

By combining REp variants with SEp variants we were able to explore a variety of distances $D$. If REp-increase-2 and SEp-increase2 are combined, for example, the distance $D$ is increased by 2 everywhere in the lattice. In this case, as well as in the case (REpdecrease-2 + SEp-decrease-2), no tubes are formed. Instead, a characteristic tangle of filaments (see Supporting Fig. 16) are formed. However, when $D$ is increased or decreased by 1 base everywhere, as in the case (REp-increase- $1+$ SEp-increase-1) where $D=22$ or (REp-decrease-1 + SEp-decrease-1) where $D=20$, normal tubes of typically small diameter form. Other combinations of REp and SEp variants that yield a value of $D=22$ or $D=20$ for example (REp + SEp-increase-2) and (REp + SE-decrease-2) also form tubes with the normal orientation of tiles. Such lattices have the same phosphate backbone as the tubes created by (REp-increase- $1+$ SEpincrease-1) and (REp-decrease-1 + SEp-increase-1) except for the positions of the nicks at the sticky ends. This difference seems minor but may be responsible for the obervation that the tubes formed by (REp + SE-decrease-2) are larger (N11-16) and appear to have 
some helicity (Supporting Fig. 16).

Note that here (and in the modelling for Supporting Figs. 1 and 2) we only explored changes to $D$ that were made uniformly thoughout the lattice. If the length to the left of SEp were $D=20$ while the length to the right of SEp were $D=22$ (as would be the case for SEp-increase-1 + REp-decrease-1), then the model predicts that, indeed, the tube diameter would change. This has not yet been attempted experimentally.

Correspondence should be addressed to Paul W. K. Rothemund (pwkrecaltech.edu).

\section{References}

(1) Srinivasan, K.; Pohl, G.; Avdalovic, N. Analytical Chemistry 1997, 69, 27982805.

(2) Hansma, H. G.; Laney, D. E. Biophysical Journal 1996, 70, 1933-1939.

(3) Bloomfi eld, V. A.; Crothers, D. M.; Tinoco, Jr., I. Nucleic Acids: Structures, Properties, and Functions; University Science Books: 2000 Page 408.

(4) Yamakawa, H.; Stockmayer, W. H. Journal of Chemical Physics 1972, 57, $2843-$ 2854.

(5) Sano, M.; Kamino, A.; Okamura, J.; Shinkai, S. Science 2001, 293, 1299-1301. 
DAE-E

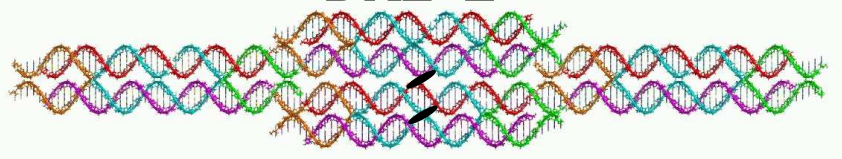

DAE-O

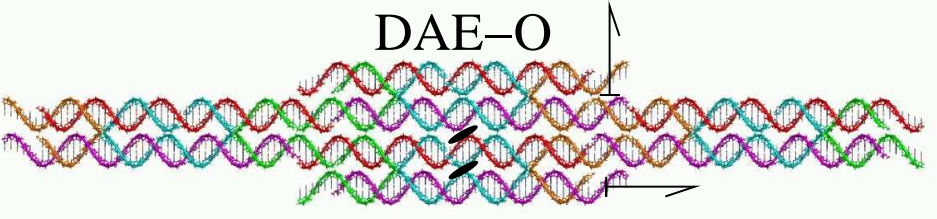

DAO-E
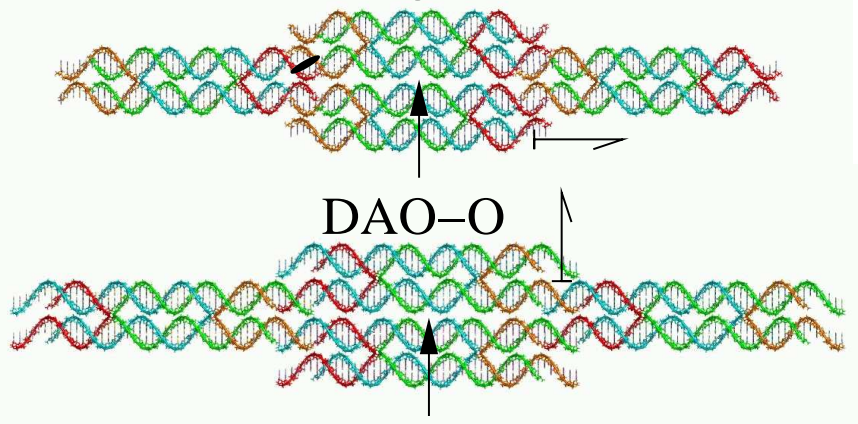

DAE-E

$\because$

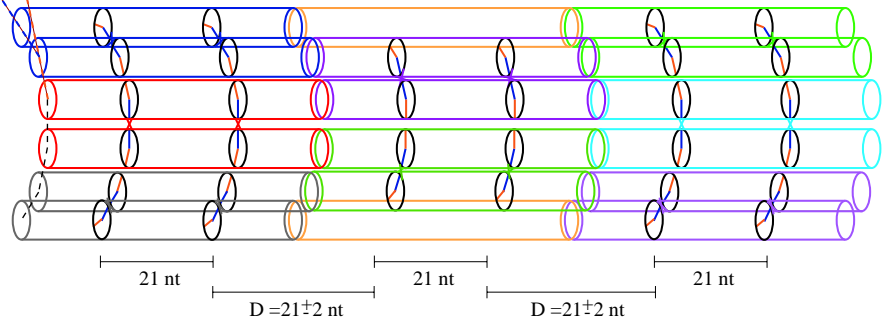

DAE-O

$\because$

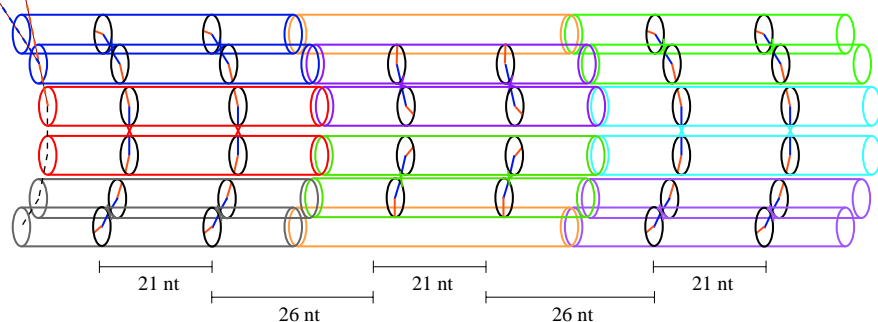

DAO-E

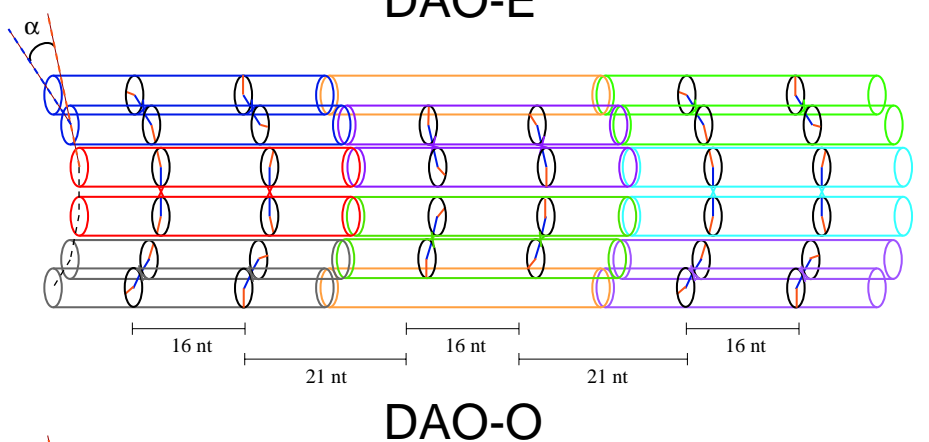

$i^{\alpha}$

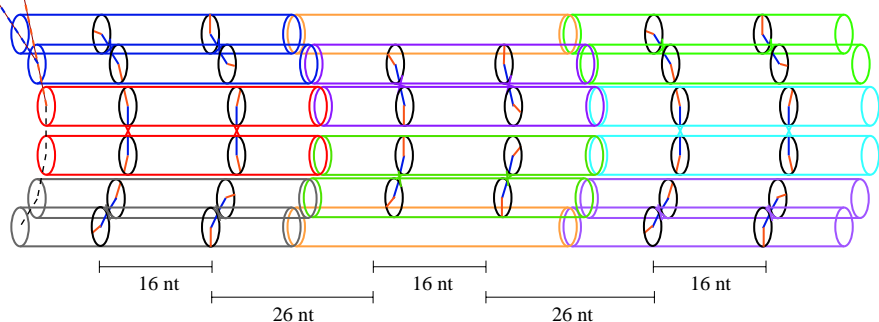

Figure 1. Top: $2 \times 2$ patches of DAE-E, DAO-E, DAE-O, and DAO-O double-crossover lattices, with rotational (black oval, axis perpendicular to the plane; arrow, axis in plane) and screw (half-arrow) symmetries of the full lattice indicated. Only the phosphate backbone and sticky-end nicks are considered; coloring of strands (denoting sequence) is not necessarily preserved by some symmetries of the phosphate backbone. Bottom: Model patches of tiles used to investigate strain balancing in curved and fht sheets. Each patch contains 8 tiles (colors) as well as two double-stranded "half-tiles" (yellow) that complete the 6 helices. Each motif is characterized by a pattern of lengths (in nucleotides) between crossovers points within the tile and between those in adjacent tiles. Here we only explore uniform lattices, where the lengths are the same at every tile position. (E.g., $D$ has the same value for all locations within a given patch.) Patch curvature, measured by the angle $\alpha$ between adjacent helices, is treated as a free parameter; we wish to estimate the strain energy as a function of $\alpha$. (An $N$-tile wide tube would have $\alpha=\frac{360^{\circ}}{2 N}$.) For each lattice motif, the relative orientation of the major and minor groove is determined at each crossover point. The exact position of the phosphate participating in the crossover is further constrained to be at the tangent point to the adjacent helix, so given $\alpha$, the position of each crossover phosphate can be determined. In an elastic rod model of DNA, ${ }^{3}$ the energy required to twist a rod by $\phi$ radians can be calculated as $\Delta G_{\text {twist }}=\frac{1}{2} C\left(\frac{\phi}{L}\right)^{2} L$ where $L$ is the length of the rod and $C=2.5 \times 10^{-19} \mathrm{erg} \mathrm{cm}$. For each helical domain between crossover points, the minimal twist $\phi$ (in addition to the relaxed B-form DNA helix twist of $34^{\circ}$ per base pair) required to meet the phosphate constraints is easily calculated. Summing over all constrained domains in the patch gives the total twist strain energy. 


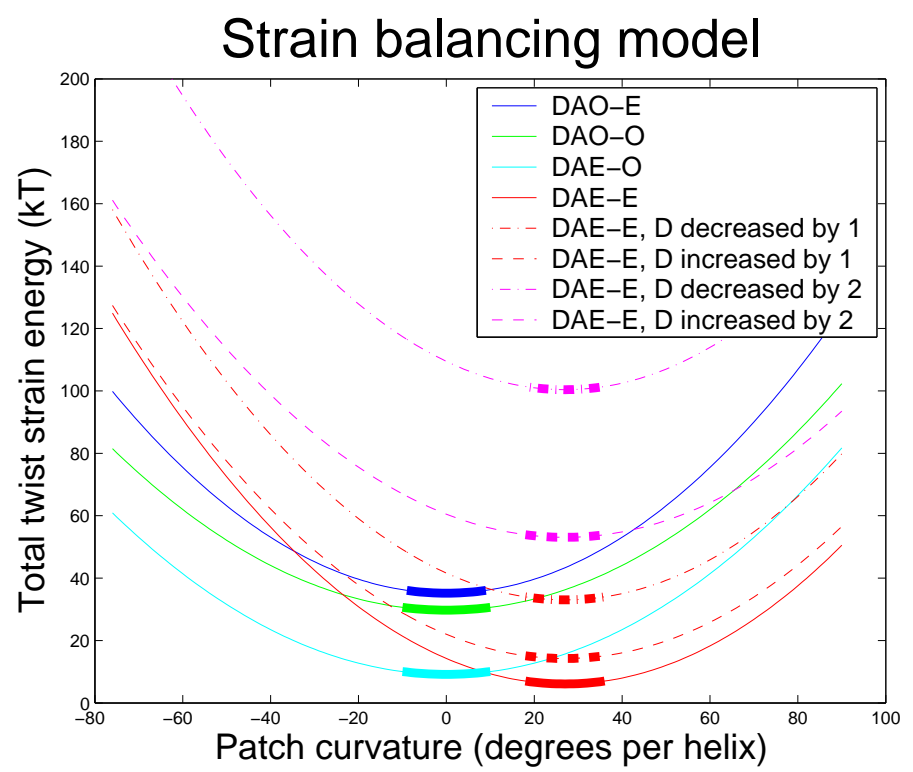

Figure 2. Plot of the total twist strain energy (per the elastic rod estimate) as a function of the patch curvature angle $\alpha$, for each of four lattice motifs (Supporting Fig. 1) and four variant spacings of the DAE-E lattice motif. Within $1 \mathrm{kT}$ of the minimum energy for each motif, the plot is shown in bold. For the DAO-E, DAO-O, and DAE-O motifs, the minimum energy is obtained by fht patches, $\alpha=0$, in agreement with the symmetry argument described in the text. Note that the value of the minimum strain energy is higher for both DAO motifs, largely because the helix domains between crossovers within the tiles are somewhat under- and over-strained. For the DAE-E motif, the minimum-energy curvature angle $\alpha$ is exactly that needed to complete the angular extent of the minor groove (assumed to be $180^{\circ}-153^{\circ}=27^{\circ}$ for the calculations shown here), in agreement with the argument given in the text. The DAE-E patches with increased or decreased $D$ have the same minimum-energy curvature angle $\alpha=27^{\circ}$, but for some even the minimum energy confi guration has substantial amounts of strain. Unlike the DAO motifs, here the strain is within helix domains containing the sticky ends, suggesting that it may not be favorable for a tile to attach to such a patch - unless the assumptions of the model are broken. (For comparison, each sticky-end hybridization is estimated to be $11 \pm 2 \mathrm{kT}$.) Fo all motifs, within $1 \mathrm{kT}$ of energy $\alpha$ can vary by $\pm 10^{\circ}$. This fi gure would, of course, change with the size of the patch. 
RES

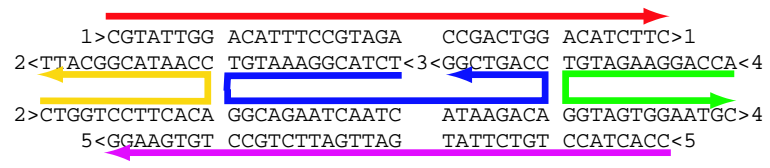

(strands RE1, RE2-EE01, RE3, RE4-DIAG, RE5)

SEd

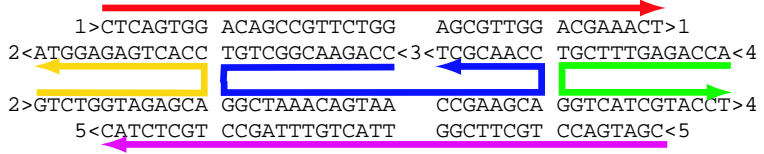

(strands SE1, SE2-DIAG, SE3, SE4-DIAG, SE5)

SEp $(1,5: h 6)$

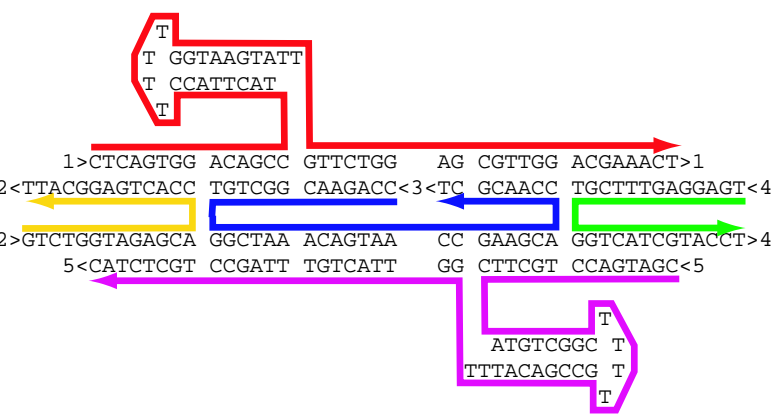

(strands SE1-h14, SE2, SE3, SE4, SE5-h14)

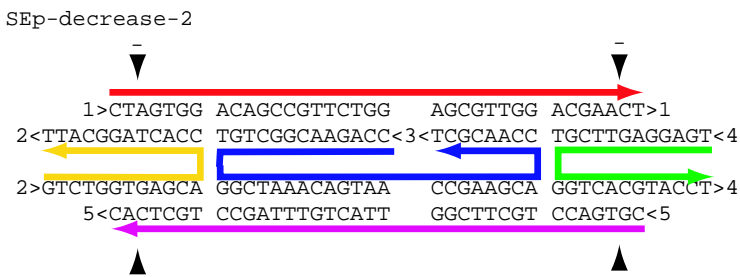

(strands SE1-D2, SE2-D2, SE3, SE4-D2, SE5-D2)

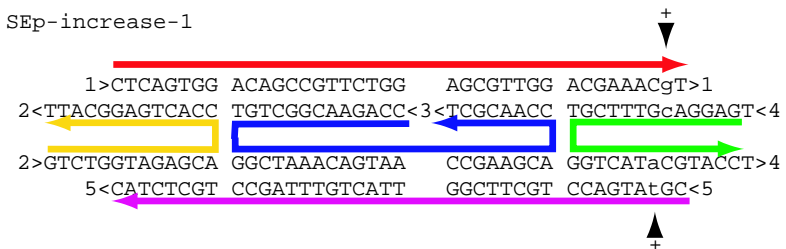

(strands SE1-A1, SE2, SE3, SE4-A2, SE5-A1)

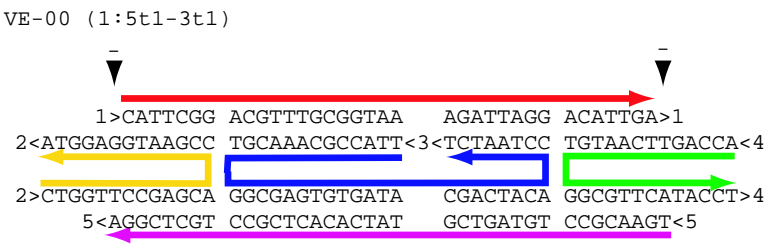

(strands VE1-5t1-3t1, VE2-EE00, VE3, VE, VE5)
SES

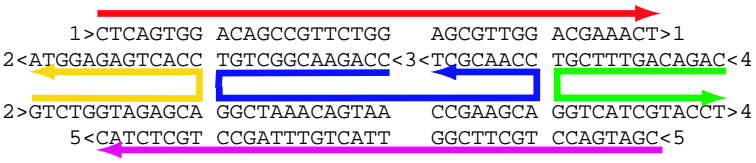

(strands SE1, SE2-DIAG, SE3, SE4-EE10, SE5)

SEn

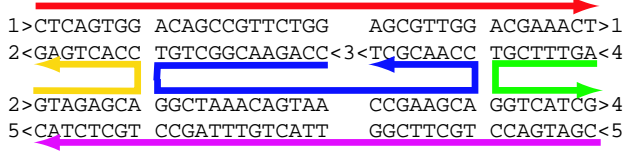

(strands SE1, SE2-NSTK, SE3, SE4-NSTK, SE5)

SEs $(1,5:$ h22-short

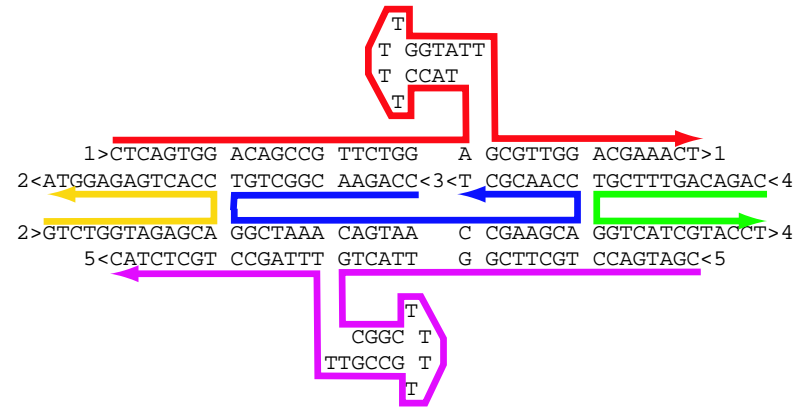

(strands SE1-h22-short, SE2-DIAG, SE3, SE4-EE10, SE5-h22-short)
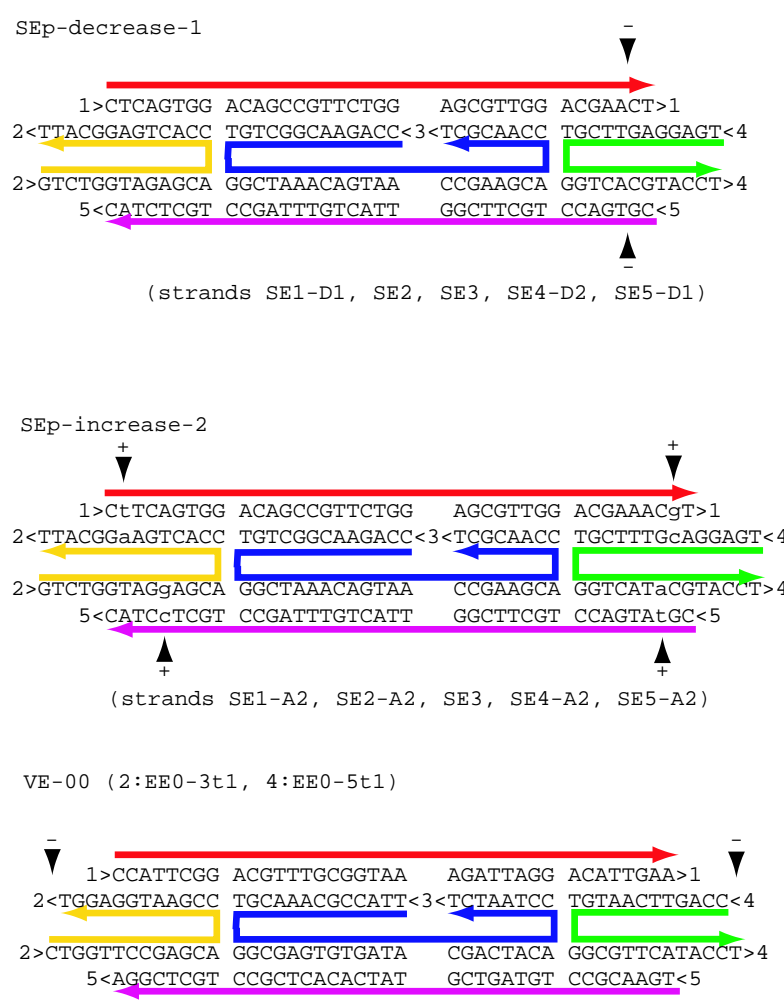

(strands VE1, VE2-EE0-3t1, VE3, VE4-EE00-5t1, VE5)

Figure 3. Schematics showing the sequences for 12 of the double crossover molecules used in this paper. Conceptually a tile is reprogrammed by taking the core sequences (all the sequences without the sticky ends) and appending a sticky end of choice. Practically, for the DAE-E double crossovers, it is done by using strands \#1, \#3, and \#5 which bear core sequences and adding new \#2 and \#4 strands bearing the sticky end sequences of choice. 


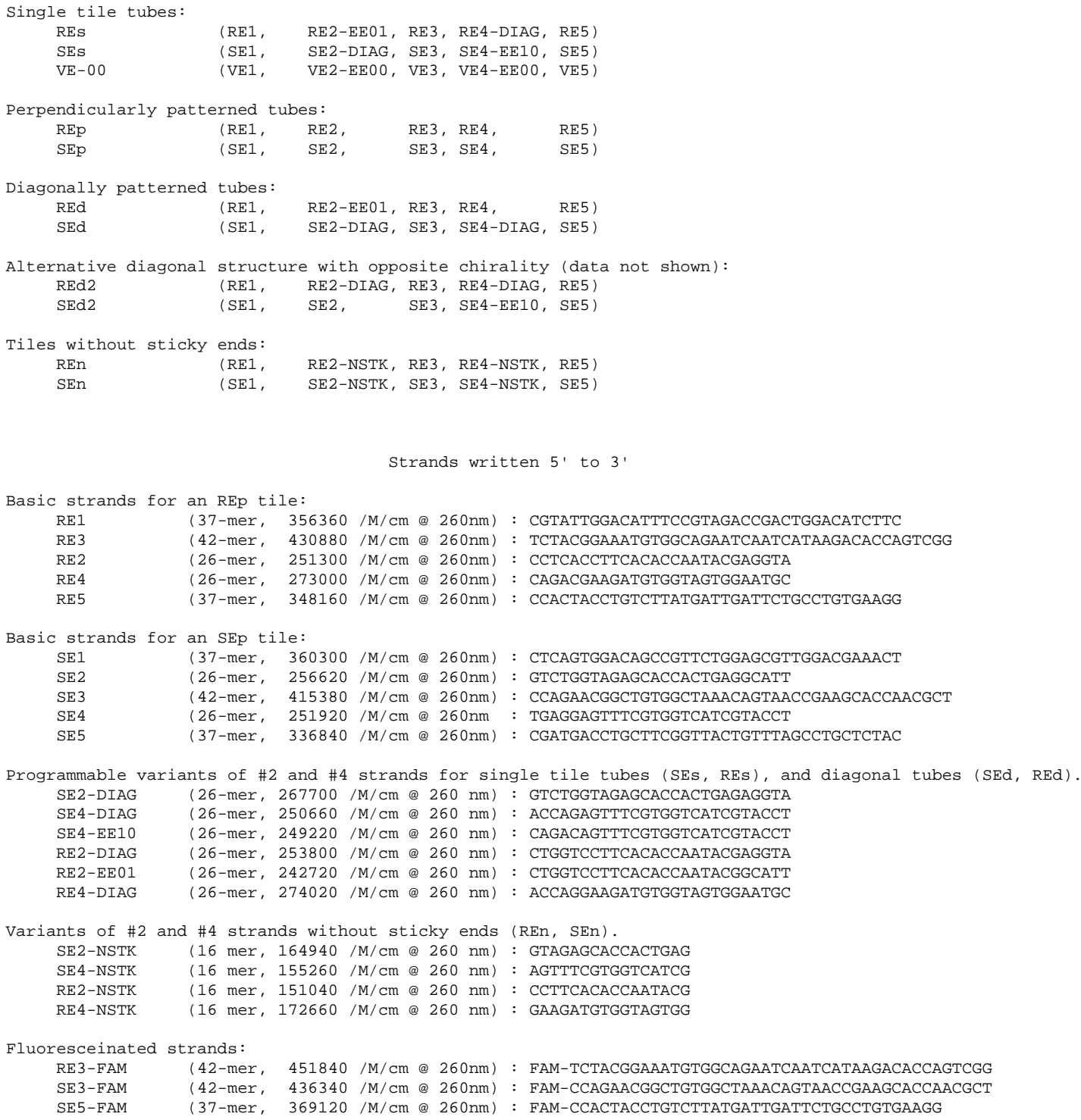

Figure 4. At top the basic strand composition of each double crossover used in this paper is shown. Below, the sequences for these double crossovers are given, as well as the FAM-modifi ed strands use in fluorescence microscopy experiments. Note that for historical reasons the pair of strands \# 2 and \# 4 used for a particular double crossover are not obvious from the name of the crossover and the names of the strands. For example, the SEs tile for single tile tubes uses an SE2-DIAG strand, and REd, for diagonally striped tubes, uses neither RE2-DIAG nor RE4-DIAG. Based on our understanding of the direction of closure of the tubes, tubes created from REd + SEd will bear a left-handed helical pattern. This can be seen by taking the diagonal pattern in Fig. 1C and rolling it up off of the page. The tiles REd2 and SEd2 bear a different choice of sticky ends that will create a right-handed diagonal tube (AFM data not shown). 
Long hairpin strands for SE5

SE5-F-7 ' h6' $^{\prime}$ (59-mer, $539360 / \mathrm{M} / \mathrm{cm}$ a $\left.260 \mathrm{~nm}\right):$ CGATGAATGTCGGCTTTTGCCGACATTTCCTGCTTCGGTTACTGTTTAGCCTGCTCTAC SE5-F-6 ' h7' (59-mer, $539700 / \mathrm{M} / \mathrm{cm}$ a $260 \mathrm{~nm})$ : CGATGACATGTCGGCTTTTGCCGACATTTCTGCTTCGGTTACTGTTTAGCCTGCTCTAC SE5-F-5 ' h8' (59-mer, $540340 / \mathrm{M} / \mathrm{cm}$ @ $260 \mathrm{~nm})$ : CGATGACCATGTCGGCTTTTGCCGACATTTTGCTTCGGTTACTGTTTAGCCTGCTCTAC SE5-F-4 ' h9' (59-mer, $540660 / \mathrm{M} / \mathrm{cm}$ @ $260 \mathrm{~nm}):$ CGATGACCTATGTCGGCTTTTGCCGACATTTGCTTCGGTTACTGTTTAGCCTGCTCTAC SE5-F-3 'h10' (59-mer, $540860 / \mathrm{M} / \mathrm{cm}$ e $260 \mathrm{~nm})$ : CGATGACCTGATGTCGGCTTTTGCCGACATTTCTTCGGTTACTGTTTAGCCTGCTCTAC SE5-F-2 'h11' (59-mer, $540340 / \mathrm{M} / \mathrm{cm}$ \& $260 \mathrm{~nm})$ : CGATGACCTGCATGTCGGCTTTTGCCGACATTTTTCGGTTACTGTTTAGCCTGCTCTAC SE5-F-1 ' $\mathrm{h} 12$ ' (59-mer, $540660 / \mathrm{M} / \mathrm{cm}$ @ $260 \mathrm{~nm})$ : CGATGACCTGCTATGTCGGCTTTTGCCGACATTTTCGGTTACTGTTTAGCCTGCTCTAC SE5-F0 'h13' (59-mer, $540660 / \mathrm{M} / \mathrm{cm}$ @ $260 \mathrm{~nm})$ : CGATGACCTGCTTATGTCGGCTTTTGCCGACATTTCGGTTACTGTTTAGCCTGCTCTAC SE5-J 'h14' (59-mer, $539060 / \mathrm{M} / \mathrm{cm}$ @ $260 \mathrm{~nm})$ : CGATGACCTGCTTCATGTCGGCTTTTGCCGACATTTGGTTACTGTTTAGCCTGCTCTAC SE5-F2 'h15' (59-mer, $539480 / \mathrm{M} / \mathrm{cm}$ @ $260 \mathrm{~nm}):$ CGATGACCTGCTTCGATGTCGGCTTTTGCCGACATTTGTTACTGTTTAGCCTGCTCTAC SE5-F3 ' 'h16' (59-mer, $539720 / \mathrm{M} / \mathrm{cm}$ e $260 \mathrm{~nm})$ : CGATGACCTGCTTCGGATGTCGGCTTTTGCCGACATTTTTACTGTTTAGCCTGCTCTAC SE5-F4 ' $\mathrm{h} 17$ ' (59-mer, $540660 / \mathrm{M} / \mathrm{cm}$ a $260 \mathrm{~nm})$ : CGATGACCTGCTTCGGTATGTCGGCTTTTGCCGACATTTTACTGTTTAGCCTGCTCTAC SE5-F5 'h18' (59-mer, $540660 / \mathrm{M} / \mathrm{cm}$ @ $260 \mathrm{~nm})$ : CGATGACCTGCTTCGGTTATGTCGGCTTTTGCCGACATTTACTGTTTAGCCTGCTCTAC SE5-F6 'h19' (59-mer, $539360 / \mathrm{M} / \mathrm{cm}$ @ $260 \mathrm{~nm}$ ) : CGATGACCTGCTTCGGTTAATGTCGGCTTTTGCCGACATTTCTGTTTAGCCTGCTCTAC SE5-FLIP 'h20' (59-mer, $540340 / \mathrm{M} / \mathrm{cm}$ @ $260 \mathrm{~nm}$ ) : CGATGACCTGCTTCGGTTACATGTCGGCTTTTGCCGACATTTTGTTTAGCCTGCTCTAC SE5-F8 'h21' (59-mer, $540660 / \mathrm{M} / \mathrm{cm}$ @ $260 \mathrm{~nm}):$ CGATGACCTGCTTCGGTTACTATGTCGGCTTTTGCCGACATTTGTTTAGCCTGCTCTAC SE5-FJ 'h22' (59-mer, $539720 / \mathrm{M} / \mathrm{cm}$ a $260 \mathrm{~nm}$ ) : CGATGACCTGCTTCGGTTACTGATGTCGGCTTTTGCCGACATTTTTTAGCCTGCTCTAC SE5-F10 'h23' (59-mer, $540660 / \mathrm{M} / \mathrm{cm}$ e $260 \mathrm{~nm})$ : CGATGACCTGCTTCGGTTACTGTATGTCGGCTTTTGCCGACATTTTTAGCCTGCTCTAC SE5-F11 'h24' (59-mer, $540660 / \mathrm{M} / \mathrm{cm}$ @ $260 \mathrm{~nm})$ : CGATGACCTGCTTCGGTTACTGTTATGTCGGCTTTTGCCGACATTTTAGCCTGCTCTAC SE5-F12 'h25' (59-mer, $540660 / \mathrm{M} / \mathrm{cm}$ @ $260 \mathrm{~nm})$ : CGATGACCTGCTTCGGTTACTGTTTATGTCGGCTTTTGCCGACATTTAGCCTGCTCTAC SE5-F13 'h26' (59-mer, $538220 / \mathrm{M} / \mathrm{cm}$ @ $260 \mathrm{~nm})$ : CGATGACCTGCTTCGGTTACTGTTTAATGTCGGCTTTTGCCGACATTTGCCTGCTCTAC SE5-F14 'h27' (59-mer, $540860 / \mathrm{M} / \mathrm{cm}$ e $260 \mathrm{~nm})$ : CGATGACCTGCTTCGGTTACTGTTTAGATGTCGGCTTTTGCCGACATTTCCTGCTCTAC SE5-F15 'h28' (59-mer, $539700 / \mathrm{M} / \mathrm{cm}$ a $260 \mathrm{~nm}$ ) : CGATGACCTGCTTCGGTTACTGTTTAGCATGTCGGCTTTTGCCGACATTTCTGCTCTAC SE5-F16 'h29' (59-mer, $540340 / \mathrm{M} / \mathrm{cm}$ @ $260 \mathrm{~nm}$ ) : CGATGACCTGCTTCGGTTACTGTTTAGCCATGTCGGCTTTTGCCGACATTTTGCTCTAC SE5-F17 'h30' (59-mer, $540660 / \mathrm{M} / \mathrm{cm}$ e $260 \mathrm{~nm}):$ CGATGACCTGCTTCGGTTACTGTTTAGCCTATGTCGGCTTTTGCCGACATTTGCTCTAC SE5-F18 'h31' (59-mer, $540860 / \mathrm{M} / \mathrm{cm}$ @ $260 \mathrm{~nm})$ : CGATGACCTGCTTCGGTTACTGTTTAGCCTGATGTCGGCTTTTGCCGACATTTCTCTAC

Short hairpin strands for $5 E 5:$

SE5-E0-short ' SE5-J-short 'h14-short' (51-mer, $457520 / \mathrm{M} / \mathrm{cm}$ e $260 \mathrm{~nm}$ ) : CGATGACCTGCTTCCGGCTTTTGCCGTTGGTTACTGTTTAGCCTGCTCTAC SE5-F2-short 'h15-short' (51-mer, $456780 / \mathrm{M} / \mathrm{cm}$ e $260 \mathrm{~nm})$ : CGATGACCTGCTTCGCGGCTTTTGCCGTTGTTACTGTTTAGCCTGCTCTAC SE5-F3-short 'h16-short' (51-mer, $457020 / \mathrm{M} / \mathrm{cm}$ e $260 \mathrm{~nm}$ ) : CGATGACCTGCTTCGGCGGCTTTTGCCGTTTTACTGTTTAGCCTGCTCTAC SE5-F4-short ' ' 117 -short' (51-mer, $458160 / \mathrm{M} / \mathrm{cm}$ a $260 \mathrm{~nm})$ : CGATGACCTGCTTCGGTCGGCTTTTGCCGTTTACTGTTTAGCCTGCTCTAC SE5-F5-short 'h18-short' (51-mer, $458160 / \mathrm{M} / \mathrm{cm}$ e $260 \mathrm{~nm})$ : CGATGACCTGCTTCGGTTCGGCTTTTGCCGTTACTGTTTAGCCTGCTCTAC SE5-F6-short 'h19-short' (51-mer, $458160 / \mathrm{M} / \mathrm{cm}$ a $260 \mathrm{~nm})$ : CGATGACCTGCTTCGGTTACGGCTTTTGCCGTTCTGTTTAGCCTGCTCTAC SE5-FLIP-short 'h20-short' (51-mer, 458800 /M/cm \& $260 \mathrm{~nm}$ ) : CGATGACCTGCTTCGGTTACCGGCTTTTGCCGTTTGTTTAGCCTGCTCTAC SE5J-F8-short 'h21-short' (51-mer, $458160 / \mathrm{M} / \mathrm{cm}$ e $260 \mathrm{~nm}$ ) : CGATGACCTGCTTCGGTTACTCGGCTTTTGCCGTTGTTTAGCCTGCTCTAC SE5-FJ-short ' h22-short' (51-mer, $457020 / \mathrm{M} / \mathrm{cm}$ a $260 \mathrm{~nm}$ ) : CGATGACCTGCTTCGGTTACTGCGGCTTTTGCCGTTTTTAGCCTGCTCTAC SE5-F10-short 'h23-short' (51-mer, $458160 / \mathrm{M} / \mathrm{cm}$ @ $260 \mathrm{~nm})$ : CGATGACCTGCTTCGGTTACTGTCGGCTTTTGCCGTTTTAGCCTGCTCTAC

Long hairpin strands for SE1:

SE1-J 'h14' (26-mer, $572120 / \mathrm{M} / \mathrm{cm}$ e $260 \mathrm{~nm})$ : CTCAGTGGACAGCCTACTTACCTTTTGGTAAGTATtGTTCTGGAGCGTTGGACGAAACT SE1-FLIP ' $\mathrm{h} 20$ ' (26-mer, $573160 / \mathrm{M} / \mathrm{cm}$ a $260 \mathrm{~nm})$ : CTCAGTGGACAGCCGTTCTGTACTTACCTTTTGGTAAGTATTGAGCGTTGGACGAAACT SE1-FJ 'h22' (26-mer, $572840 / \mathrm{M} / \mathrm{cm}$ @ $260 \mathrm{~nm}):$ CTCAGTGGACAGCCGTTCTGGATACTTACCTTTTGGTAAGTATTGCGTTGGACGAAACT

Long hairpins for RE tile, both \#1 and \#5 strands:

RE1J 'h14' (59-mer, $553620 / \mathrm{M} / \mathrm{cm}$ @ $260 \mathrm{~nm}$ ) : CGTATTGGACATTTGCTCAGCGTTTTCGCTGAGCTTCCGTAGACCGACTGGACATCTTC RE1-FJ ' 'h22' (59 mer, $554260 / \mathrm{M} / \mathrm{cm}$ @ $260 \mathrm{~nm})$ : CGTATTGGACATTTCCGTAGACGCTCAGCGTTTTCGCTGAGCTTCGACTGGACATCTTC RE5J ' 'h14' (59-mer, $549780 / \mathrm{M} / \mathrm{cm}$ a $260 \mathrm{~nm}):$ CCACTACCTGTCTTCTTGCGACTTTTGTCGCAAGTTATGATTGATTCTGCCTGTGAAGG RE5-FJ 'h22' (59 mer, $549200 / \mathrm{M} / \mathrm{cm}$ \& $260 \mathrm{~nm}$ ) : CCACTACCTGTCTTATGATtGACTTGCGACTTtTGTCGCAAGTTTTCTGCCTGTGAAGG

Biotinylated strands for streptavidin binding assay, B = biotinylated $\mathrm{T}$ from IDT DNA technologies: SE5-F-7-T-bio ' b6' (38-mer, $345940 / \mathrm{M} / \mathrm{cm}$ \& $260 \mathrm{~nm})$ : CGATGABCCTGCTTCGGTTACTGTTTAGCCTGCTCTAC SE5-F-6-T-bio ' b7' (38-mer, $344720 / \mathrm{M} / \mathrm{cm}$ a $260 \mathrm{~nm}$ ) : CGATGACBCTGCTTCGGTTACTGTTTAGCCTGCTCTAC SE5-F-5-T-bio ' b8' (38-mer, $345360 / \mathrm{M} / \mathrm{cm}$ @ $260 \mathrm{~nm}$ ) : CGATGACCBTGCTTCGGTTACTGTTTAGCCTGCTCTAC SE5-F-4-T-bio ' b9' (38-mer, $345360 / \mathrm{M} / \mathrm{cm}$ \& $260 \mathrm{~nm})$ : CGATGACCTBGCTTCGGTTACTGTTTAGCCTGCTCTAC SE5-F-3-T-bio 'b10' (38-mer, $346500 / \mathrm{M} / \mathrm{cm}$ @ $260 \mathrm{~nm}$ ) : CGATGACCTGBCTTCGGTTACTGTTTAGCCTGCTCTAC SE5-F-2-T-bio 'bl1' (38-mer, $345360 / \mathrm{M} / \mathrm{cm}$ a $260 \mathrm{~nm})$ : CGATGACCTGCBTTCGGTTACTGTTTAGCCTGCTCTAC SE5-F-1-T-bio 'b12' (38-mer, $345360 / \mathrm{M} / \mathrm{cm}$ a $260 \mathrm{~nm})$ : CGATGACCTGCTBTCGGTTACTGTTTAGCCTGCTCTAC SE5-F0-T-bio 'b13' (38-mer, $345360 / \mathrm{M} / \mathrm{cm}$ e $260 \mathrm{~nm}$ ) : CGATGACCTGCTTBCGGTTACTGTTTAGCCTGCTCTAC SE5-F1-T-bio 'b14' (38-mer, $344080 / \mathrm{M} / \mathrm{cm}$ @ $260 \mathrm{~nm})$ : CGATGACCTGCTTCBGGTTACTGTTTAGCCTGCTCTAC SE5-F2-T-bio 'b15' (38-mer, $345120 / \mathrm{M} / \mathrm{cm}$ e $260 \mathrm{~nm})$ : CGATGACCTGCTTCGBGTTACTGTTTAGCCTGCTCTAC SE5-F3-T-bio 'b16' (38-mer, $345360 / \mathrm{M} / \mathrm{cm}$ a $260 \mathrm{~nm})$ : CGATGACCTGCTTCGGBTTACTGTTTAGCCTGCTCTAC SE5-F4-T-bio 'b17' (38-mer, $345360 / \mathrm{M} / \mathrm{cm}$ \& $260 \mathrm{~nm})$ : CGATGACCTGCTTCGGTBTACTGTTTAGCCTGCTCTAC SE5-F5-T-bio 'b18' (38-mer, $345360 / \mathrm{M} / \mathrm{cm}$ @ $260 \mathrm{~nm})$ : CGATGACCTGCTTCGGTTBACTGTTTAGCCTGCTCTAC SE5-F6-T-bio 'b19' (38-mer, $345940 / \mathrm{M} / \mathrm{cm}$ @ $260 \mathrm{~nm})$ : CGATGACCTGCTTCGGTTABCTGTTTAGCCTGCTCTAC SE5-F7-T-bio 'b20' (38-mer, $345360 / \mathrm{M} / \mathrm{cm}$ @ $260 \mathrm{~nm}$ ) : CGATGACCTGCTTCGGTTACBTGTTTAGCCTGCTCTAC SE5-F8-T-bio 'b21' (38-mer, $345360 / \mathrm{M} / \mathrm{cm}$ @ $260 \mathrm{~nm})$ : CGATGACCTGCTTCGGTTACTBGTTTAGCCTGCTCTAC SE5-F9-T-bio 'b22' (38-mer, $345360 / \mathrm{M} / \mathrm{cm}$ @ $260 \mathrm{~nm}$ ) : CGATGACCTGCTTCGGTTACTGBTTTAGCCTGCTCTAC SE5-F10-T-bio 'b23' (38-mer, $345360 / \mathrm{M} / \mathrm{cm}$ @ $260 \mathrm{~nm})$ : CGATGACCTGCTTCGGTTACTGTBTTAGCCTGCTCTAC SE5-F11-T-bio 'b24' (38-mer, $345360 / \mathrm{M} / \mathrm{cm}$ @ $260 \mathrm{~nm})$ : CGATGACCTGCTTCGGTTACTGTTBTAGCCTGCTCTAC SE5-F12-T-bio 'b25' (38-mer, $345360 / \mathrm{M} / \mathrm{cm}$ @ $260 \mathrm{~nm})$ : CGATGACCTGCTTCGGTTACTGTTTBAGCCTGCTCTAC SE5-F13-T-bio 'b26' (38-mer, $344800 / \mathrm{M} / \mathrm{cm}$ a $260 \mathrm{~nm}$ ) : CGATGACCTGCTTCGGTTACTGTTTABGCCTGCTCTAC SE5-F14-T-bio 'b27' (38-mer, $346500 / \mathrm{M} / \mathrm{cm}$ \& $260 \mathrm{~nm}$ ) : CGATGACCTGCTTCGGTTACTGTTTAGBCCTGCTCTAC SE5-F15-T-bio 'b28' (38-mer, $344720 / \mathrm{M} / \mathrm{cm}$ a $260 \mathrm{~nm}$ ) : CGATGACCTGCTTCGGTTACTGTTTAGCBCTGCTCTAC SE5-F16-T-bio 'b29' (38-mer, $345360 / \mathrm{M} / \mathrm{cm} @ 260 \mathrm{~nm}$ ) : CGATGACCTGCTTCGGTTACTGTTTAGCCBTGCTCTAC SE5-F17-T-bio 'b30' (38-mer, $345360 / \mathrm{M} / \mathrm{cm}$ \& $260 \mathrm{~nm}$ ) : CGATGACCTGCTTCGGTTACTGTTTAGCCTBGCTCTAC SE5-F18-T-bio 'b31' (38-mer, $346500 / \mathrm{M} / \mathrm{cm}$ \& $260 \mathrm{~nm}$ ) : CGATGACCTGCTTCGGTTACTGTTTAGCCTGBCTCTAC

Figure 5. Sequences used in the hairpin insertion and biotin insertion experiments as well as other miscellaneous hairpins. Biotins are attached to the inserted 'T' by a 6-carbon linker. The sequence names, as ordered and archived in the lab, are given at the beginning of each line; these names differ from the aliases used in this paper which appear, in quotes, to the right of a sequences given name. 


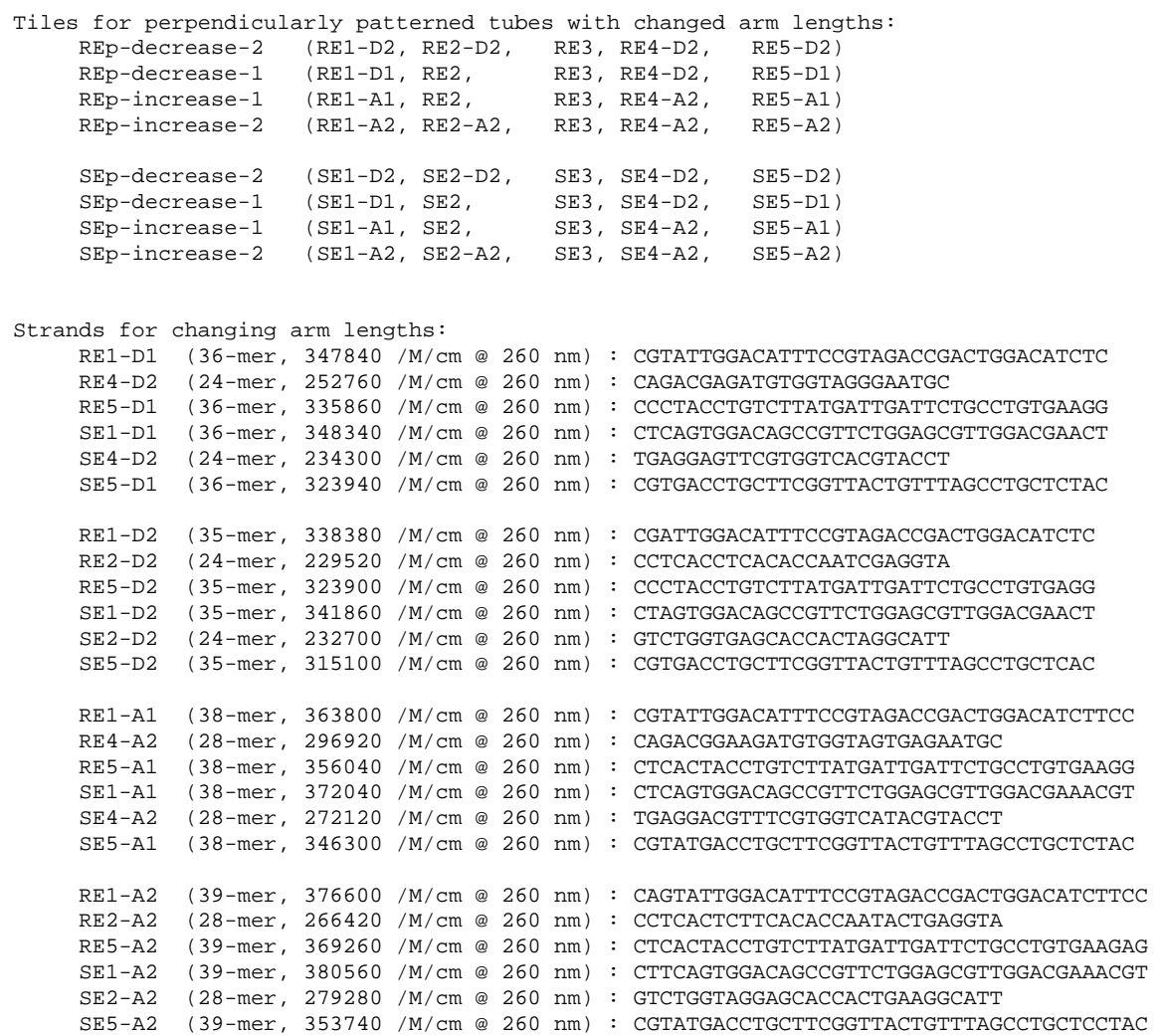

Sequences for weakening sticky ends or stacking:

SE1-5t1-3t1 (35 mer, $345960 / \mathrm{M} / \mathrm{cm}$ ) : TCAGTGGACAGCCGTTCTGGAGCGTTGGACGAAAC

SE2-DIAG-5t 1 (25 mer, $255960 / \mathrm{M} / \mathrm{cm}):$ TCTGGTAGAGCACCACTGAGAGGTA

SE2-DIAG-3t 1 (25 mer, $252840 / \mathrm{M} / \mathrm{cm}):$ GTCTGGTAGAGCACCACTGAGAGGT

SE4-EE10-5t1 (25 mer, $243220 / \mathrm{M} / \mathrm{cm})$ : AGACAGTTTCGTGGTCATCGTACCT

SE4-EE10-3t 1 (25 mer, $241500 / \mathrm{M} / \mathrm{cm}):$ CAGACAGTTTCGTGGTCATCGTACC

SE5-5t1-3t 1 (35 mer, $324220 / \mathrm{M} / \mathrm{cm}):$ GATGACCTGCTTCGGTTACTGTTTAGCCTGCTCTA

RE1-5t1-3t1 (35 mer, $342140 / \mathrm{M} / \mathrm{cm})$ : GTATTGGACATTTCCGTAGACCGACTGGACATCTT

RE2-EE01-5t 1 (25 mer, $236100 / \mathrm{M} / \mathrm{cm})$ : TGGTCCTTCACACCAATACGGCATT

RE2-EE01-3t 1 (25 mer, $234200 / \mathrm{M} / \mathrm{cm})$ : CTGGTCCTTCACACCAATACGGCAT

RE4-DIAG-5t 1 (25 mer, $260280 / \mathrm{M} / \mathrm{cm})$ : CCAGGAAGATGTGGTAGTGGAATGC

RE4-DIAG-3t 1 (25 mer, $267800 / \mathrm{M} / \mathrm{cm})$ : ACCAGGAAGATGTGGTAGTGGAATG

RE5-5t1-3t 1 (35 mer, $330020 / \mathrm{M} / \mathrm{cm})$ : CACTACCTGTCTTATGATTGATTCTGCCTGTGAAG

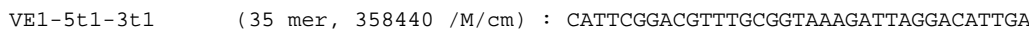

VE5-5t1-3t 1 (35 mer, $327220 / \mathrm{M} / \mathrm{cm}):$ GAACGCCTGTAGTCGTATCACACTCGCCTGCTCGG

VE2-EE00-3t 1 (25 mer, $245680 / \mathrm{M} / \mathrm{cm}) \quad:$ CTGGTTCCGAGCACCGAATGGAGGT

VE4-EE00-5t 1 (25 mer, $236060 / \mathrm{M} / \mathrm{cm}):$ CCAGTTCAATGTGGCGTTCATACCT

Figure 6. Tiles with changed arm lengths and the sequences used to change arm length, stacking interactions, or sticky-end length. Tiles used for the stacking interaction and sticky-end length experiments may be derived from the table in Supporting Fig. 17. 

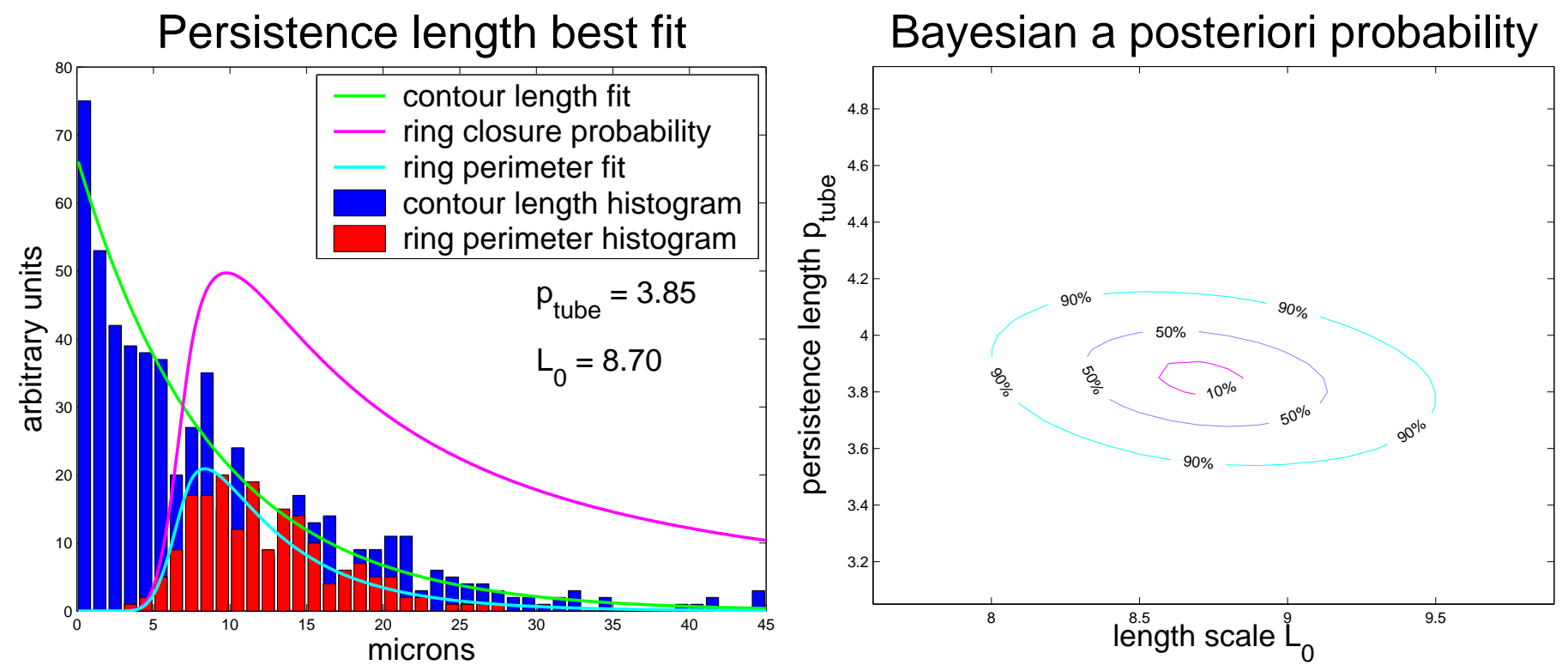

Figure 7. Left: Best simultaneous fi t (by Bayesian a posteriori probability) for tube contour length distribution $W(L) \propto e^{-L / L_{0}}$ and ring perimeter distribution $R(L) \propto W(L) G\left(L ; p_{\text {tube }}\right)$, where the ring closure probability 4 ,5 is approximated by $G(L ; p)=496.51 r^{-1} e^{-7.0266 / r}(1-0.81242 r)$ for $r<0.96093$ and $G(L ; p)=0.32992 r^{-1.5}\left(1-0.625 r^{-1}-0.12344 r^{-2}\right)$ for $r>0.96093$ and $r=\frac{L}{2 p}$. An assumption of this approach is that ring formation is slow and does not signifi cantly affect the distribution of tube contour lengths from which rings form; that rings constitute less than $2 \%$ of observed structures is consistent with this assumption. Although some ring formation could have occurred during maturation, resulting in ring closure from a different contour length distribution, this method is relatively insensitive to the contour length distribution. Changes of up to a factor of 2 in mean length would not affect the inferred $p_{t u b e}$ by more than $0.7 \mu \mathrm{m}$. Right: Contour plot showing the Bayesian a posteriori probability as a function of the two model parameters, $L_{0}$ and $p_{\text {tube }}$. Thus, $3.5<p_{\text {tube }}<4.2$ with over $90 \%$ probability, given the model assumptions. Bayesian analysis was performed by calculating, for given model parameters, the a posteriori probability $\operatorname{Pr}($ parameters $\mid$ data $)=\operatorname{Pr}($ data $\mid$ parameters $) \cdot \operatorname{Pr}($ parameters $) / \operatorname{Pr}($ data $)$, where the a priori $\operatorname{Pr}($ parameters $)$ is taken to be uniform and $\operatorname{Pr}($ data $)$ is estimated as a normalizing factor by summing $\operatorname{Pr}($ parameters $\mid$ data $)$ over a wide range of model parameters $\left(1 \leq p_{\text {tube }} \leq 10,1 \leq L_{0} \leq 40\right)$. $\operatorname{Pr}($ data|parameters $)$ was computed by assuming the contour length and ring perimeter histograms were generated by independent, indentically-distributed samples from $W(L)$ and $R(L)$ respectively. Thus, if the histogram data is $\left\{\left(L_{i}, W_{i}, R_{i}\right)\right\}_{i=1}^{N}$, with $N_{W}=\sum_{i} W_{i}$ and $N_{R}=\sum_{i} R_{i}$, then $\operatorname{Pr}($ data|parameters $\left.)=\left(\begin{array}{c}N_{W} \\ W_{1}, W_{2}, \ldots, W_{N}\end{array}\right) \prod_{i}\left[W\left(L_{i}\right)\right]^{W_{i}}\right) \cdot\left(\left(\begin{array}{c}N_{R} \\ R_{1}, R_{2}, \ldots, R_{N}\end{array}\right) \prod_{i}\left[R\left(L_{i}\right)\right]^{R_{i}}\right)$. This is more conveniently calculated as the likelihood: $\ln \operatorname{Pr}($ data|parameters $)=K+\sum_{i}\left[W_{i} \cdot \ln W\left(L_{i}\right)\right]+\left[R_{i} \cdot \ln R\left(L_{i}\right)\right]$, where $K$ is a constant independent of model parameters and is thus absorbed during normalization. Because the histogram data for $1 \mu \mathrm{m}$ contour length had experimental diffi culties, we also performed this analysis with that data omitted; indistinguishable results were obtained. 


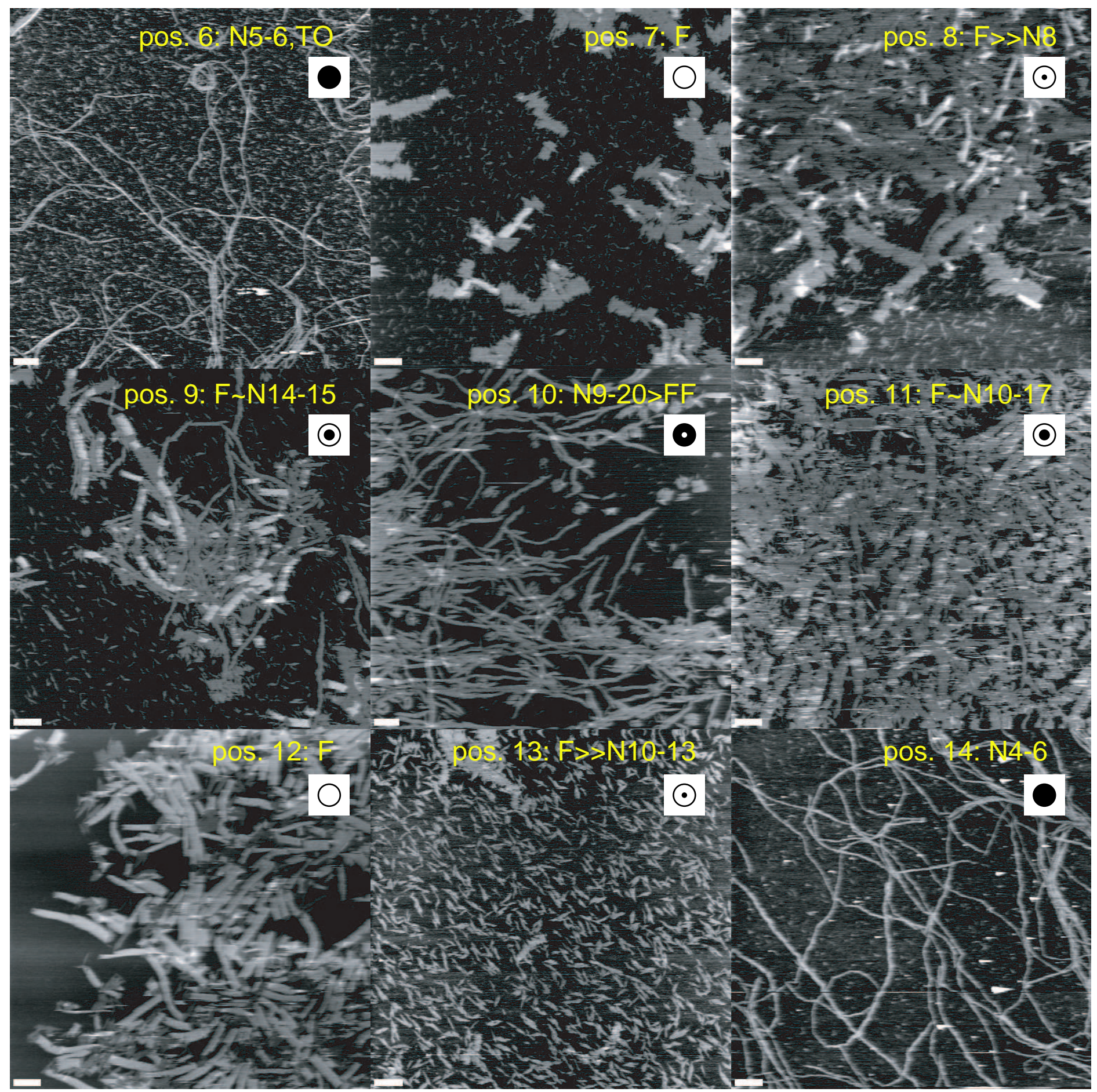

Figure 8. Representative atomic force micrographs show the results of the hairpin insertion experiments. Shown are positions 6-14 for SEs(5:hX). Circular symbols denote the extent of normal to flpped character of the samples shown and are the same as in Fig. 4. 


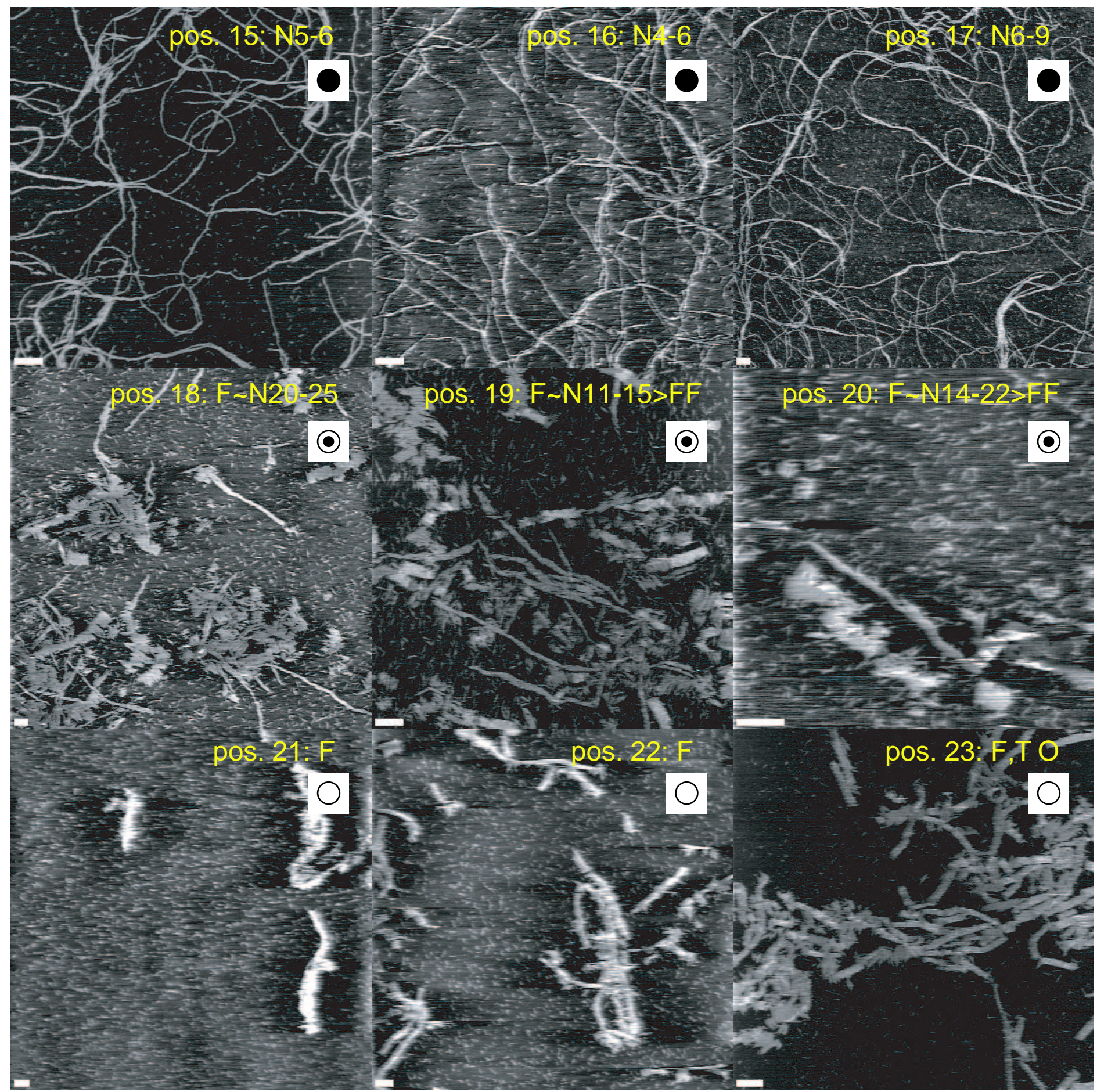

Figure 9. Representative atomic force micrographs show the results of the hairpin insertion experiments. Shown are positions 15-23 for SEs(5:hX). Circular symbols denote the extent of normal to flpped character of the samples shown and are the same as in Fig. 4. 


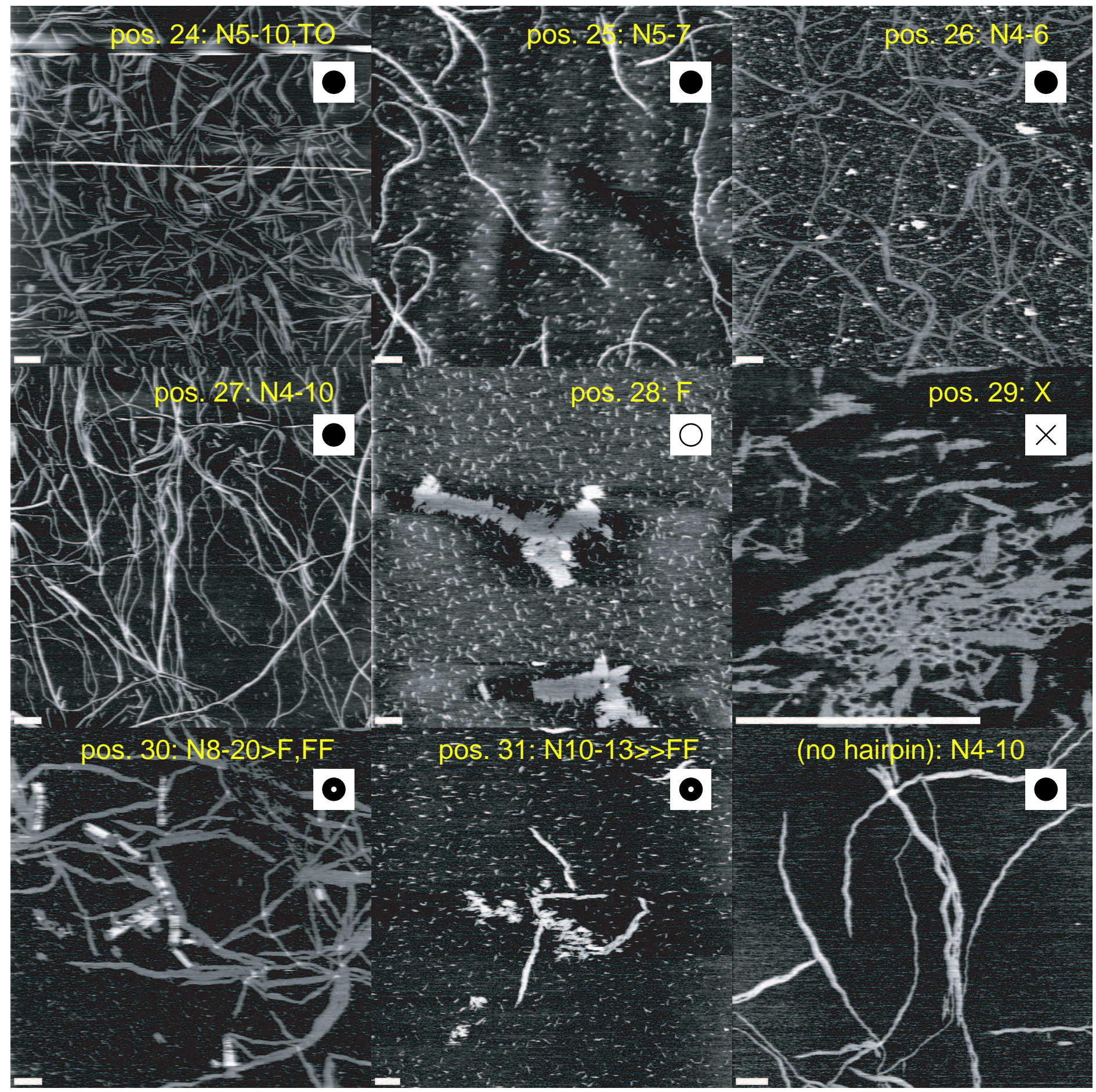

Figure 10. Representative atomic force micrographs show the results of the hairpin insertion experiments. Shown are positions 24-31 for SEs(5:hX). Circular symbols denote the extent of normal to fipped character of the samples shown and are the same as in Fig. 4. 

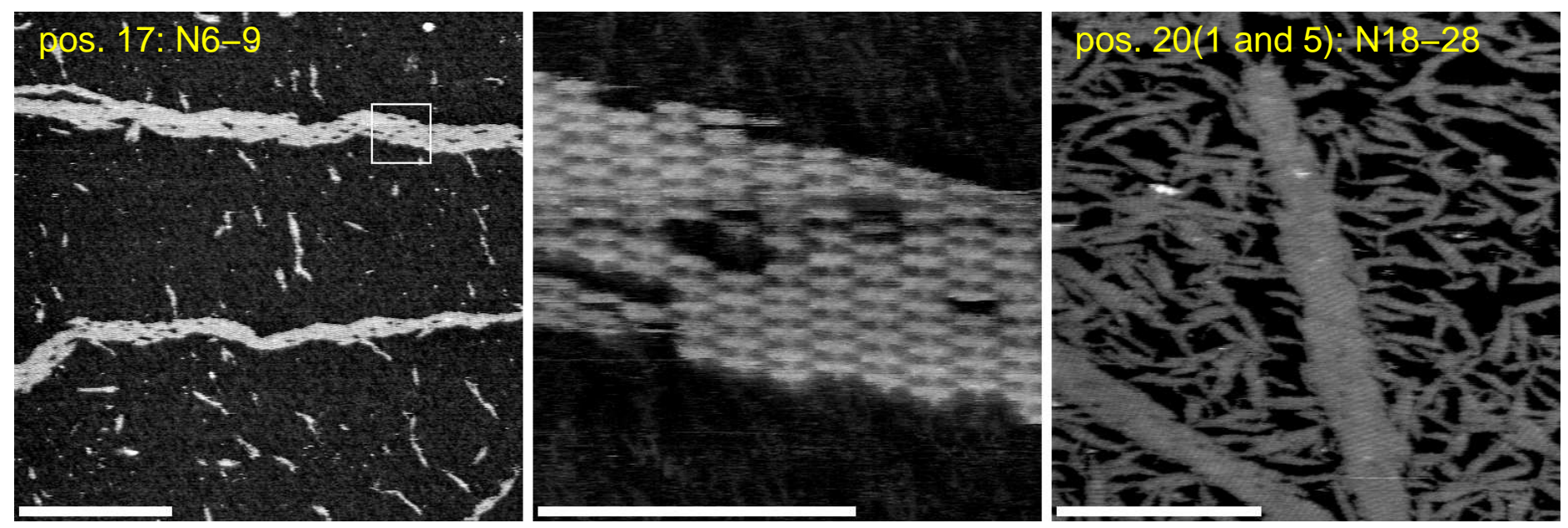

Figure 11. More hairpin-insertion images. (A, left) Often a single linear structure appears to fray or split in ways that suggest it may have been a rope or tangle of two or more tubes that opened on the surface and subsequently fused together. Here tubes are SEs(5:h17). Scale bar, 100 nm. (B, middle) High resolution image of the boxed area in (A) where hairpins can be seen. Hairpins may be seen on the bottom edges of tiles but not on the top. Scale bar, $500 \mathrm{~nm}$. (C, right) The double-hairpin single tile tube SEs $(1,5: \mathrm{h} 20)$ produced unusually wide tubes with the tiles of the normal orientation. Scale bar, $500 \mathrm{~nm}$.
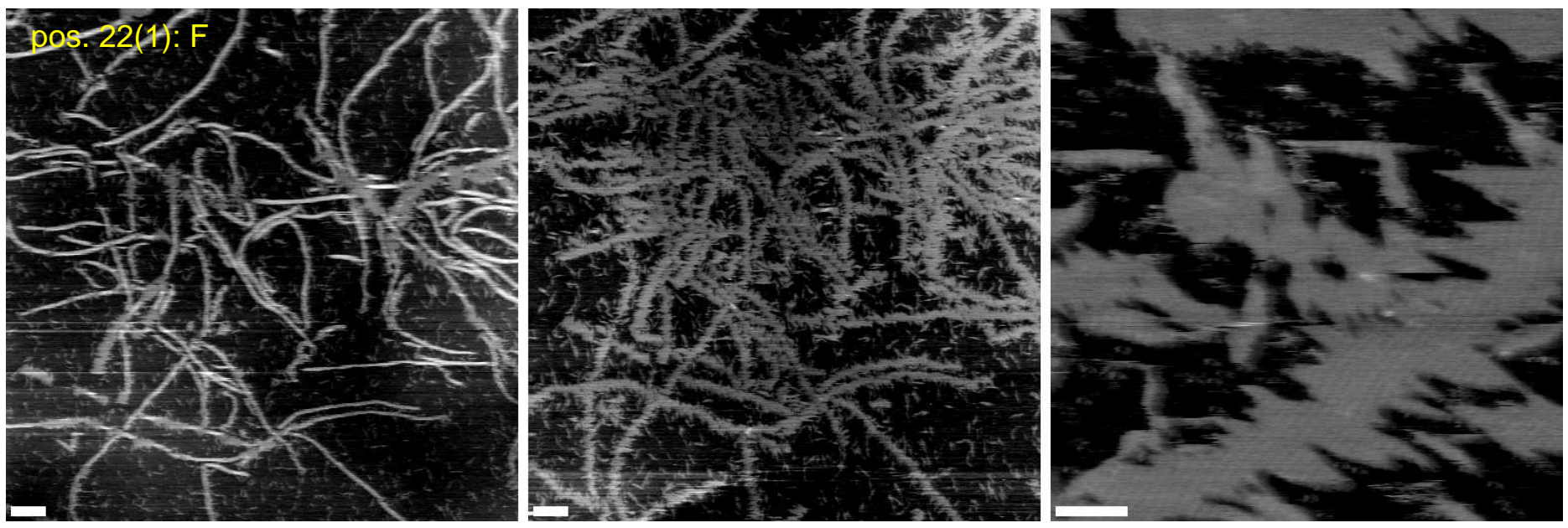

Figure 12. AFM of SEs(1:h22) fipped tubes. This modifi cation, a hairpin at position 22 on the \# 1 strand, was used to created all the fipped biotinylated tubes SEs(1:h22,3:FAM,5:bX) used the the streptaviding binding experiment. (left) Flipped tubes before opening. Scale bar, $500 \mathrm{~nm}$. (middle) Same area as at left, but after several scans have opened the tubes. Scale bar, $500 \mathrm{~nm}$. (right) A zoom of the middle panel shows that the circumference of these tubes was $\approx$ 9-11 tiles and $\approx 143 \mathrm{~nm}$. The diameter was thus $\approx 45 \mathrm{~nm}$, large enough to admit streptavidin-Cy3. Scale bar, $100 \mathrm{~nm}$. 


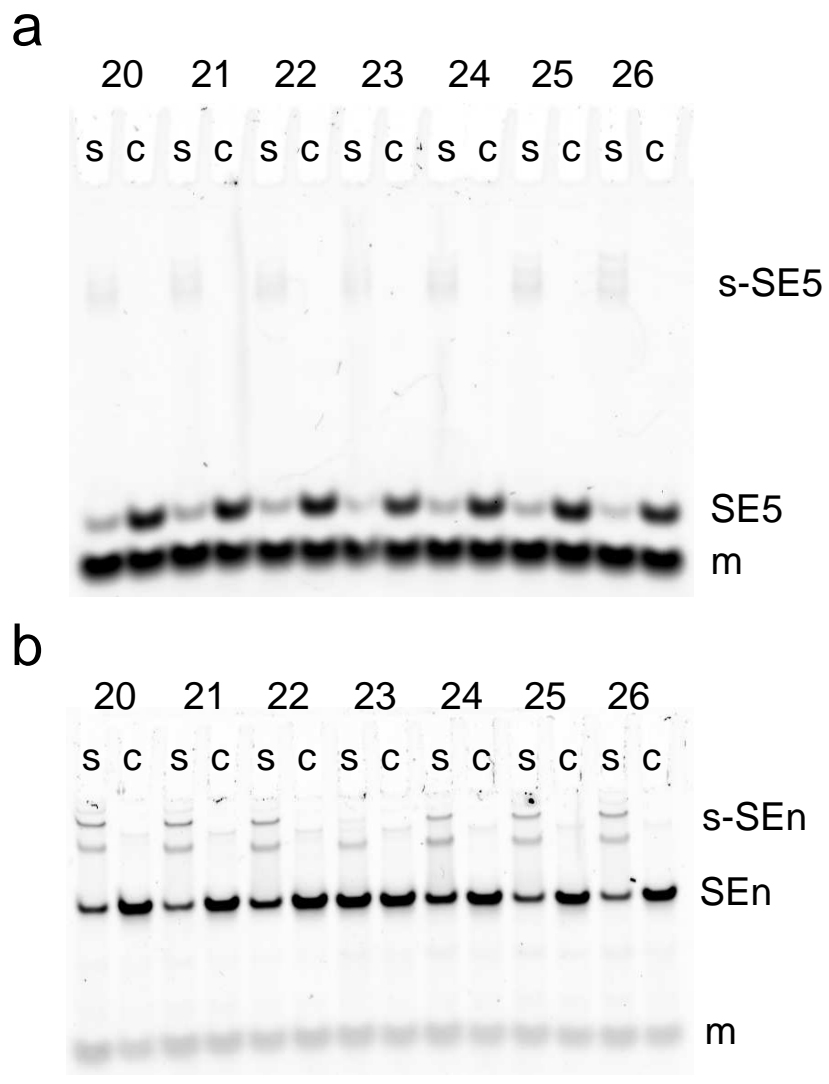

Figure 13. Examples of non-denaturing gels used to test biotin functionality and background streptavidin labelling of tiles as using procedures described in Methods. (a) For each of the strands SE5-bX, a gel shift assay was performed to determine the extent of biotin labelling. Shown are positions $X=20-26$. Control lanes marked ' $c$ ' contain the 38-mer strands SE5-bX in the upper band mixed with a 26-mer marker strand ' $m$ ', RE4-DIAG, in the lower band. Lanes marked 's' contain the latter mixture with an excess of streptavidin-Cy3 added. SE5-bX and the marker were visualized by Sybr Green I staining and quantitated: $S$ refers to the measured amount of SE5-bX in the 's' lane, $S M$, the amount of marker in the 's' lane, $C$ the amount of SE5-bX in the 'c' lane and $C M$ the amount of marker in the 'c' lane. The fraction of SE5-bX with functional biotin was computed to be $1-\frac{S / S M}{C / C M}$. This assay indicated that the effi ciency of biotin labelling of the strands ranged from $52 \%$ to $82 \%$. In principle these numbers could be used to normalize experiments testing the binding of streptavidin to biotinylated tiles or tubes (bearing the relevant SE5-bX strand). In practice we found such normalizations made the measurements noiser and thus we performed no normalization on these later experiments. (b) For each of the tiles $\operatorname{SEn}(5: \mathrm{b} X)$, a gel shift assay was perfomed to determine the background profi le of streptavidin binding to tiles outside of the context of a lattice or tube. Shown are positions $X=20-26$. Control lanes marked 'c' contain the tile SEn(5:bX) in the upper band mixed with a 26-mer marker strand ' $m$ ', RE4-DIAG, in the lower band. Lanes marked 's' contain the latter mixture with an excess of streptavidin-Cy3 added. $\mathrm{SEn}(5: \mathrm{b} X)$ and the marker were visualized by Sybr Green I staining and quantitated: $S$ refers to the measured amount of $\mathrm{SEn}(5: \mathrm{b} X)$ in the 's' lane, $S M$, the amount of marker in the 's' lane, $C$ the amount of $\operatorname{SEn}(5: \mathrm{b} X)$ in the 'c' lane and $C M$ the amount of marker in the 'c' lane. The accessibility of biotin for streptavidin binding on a tile $\operatorname{SEn}(5: \mathrm{b} X)$ was computed to be $1-\frac{S / S M}{C / C M}$. In particular such gels were meant to isolate the effect of the intramolecular contact between helices on the effi ciency of streptavidin binding. Because the variation in streptavidin binding for biotin positions on the accessible portions of a tile is lower than the variation observed in experiments measuring the fraction of functionally biotinylated SE5-bX strands from (a) we, did not normalize this data. We suspect that the variation in (a) may be due not to a variation in the biotinylation of the oligos but rather to a position-dependent change in accessibility of the biotin based on the secondary structure of the SE5-bX oligo. 

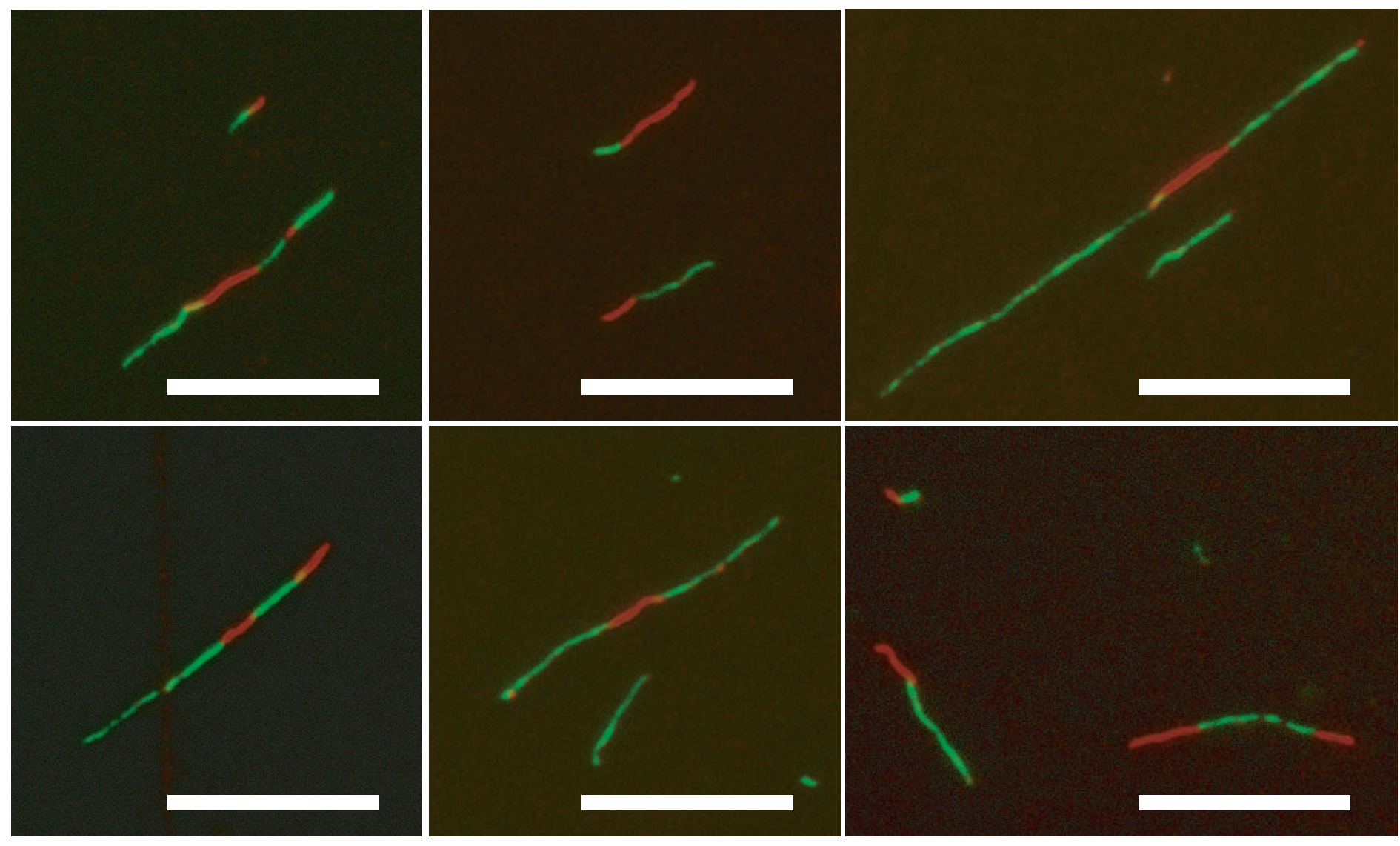

Figure 14. Here, to create hybrid tubes, $25 \mu \mathrm{l}$ of SEs(3:FAM, 5:b15) tubes and $25 \mu \mathrm{l}$ of SEs(5:b22) tubes at $200 \mathrm{nM}$ were annealed separately, then mixed and sonicated in an ultrasonic water bath (Branson 2210, $47 \mathrm{KHz}$.) for 30 seconds before labelling with streptavidin-Cy3. Without sonication, the frequency of end-joining between the two types of tubes is observed to be quite small, $\approx 0.5 \%$ of tubes were mult-colored. Immediately after sonication, a similarlyprepared sample contained only small tube fragments $(\approx 2-4 \mu \mathrm{m})$. Several hours after sonication $\approx 5-10 \%$ of tubes were multi-colored, many with multiple domains, as seen above. (Tubes are stretched and immobilized on untreated glass, the contours they assume are not indicative of their persistence length.) It is not known whether sonication merely increases the number of free ends available for joining or causes tubes to shear in a manner that makes them more likely to join. These experiments show that tubes with multiple chemical characters may be fused into single tubes. Scale bars $10 \mu \mathrm{m}$.
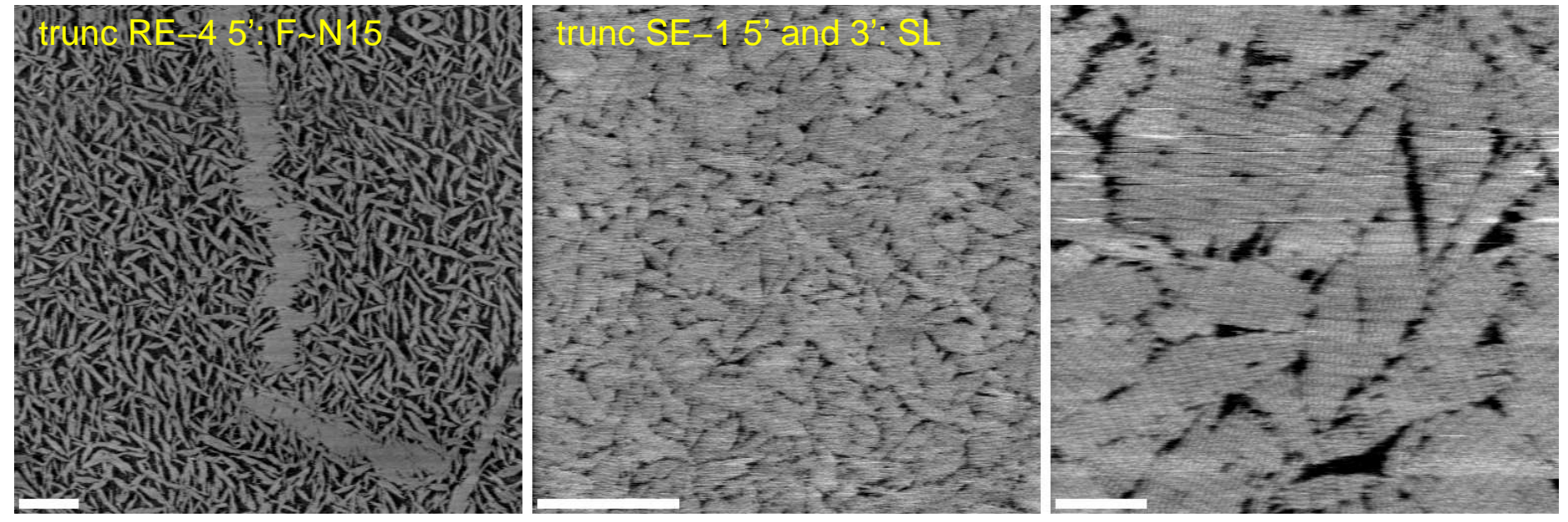

Figure 15. (left) A single sticky end truncation that yields fipped tubes. Scale bar, $500 \mathrm{~nm}$. (middle) Most trunctations, including this double truncation of the $5^{\prime}$ and $3^{\prime}$ nucleotides of SE-1, do not yield any kind of tube. The small lattice fragments seen here may well have assembled on the mica at the time of sample preparation. Scale bar, $500 \mathrm{~nm}$. (right) Zoom of middle panel in which tiles are resolved. Scale bar, $100 \mathrm{~nm}$. 

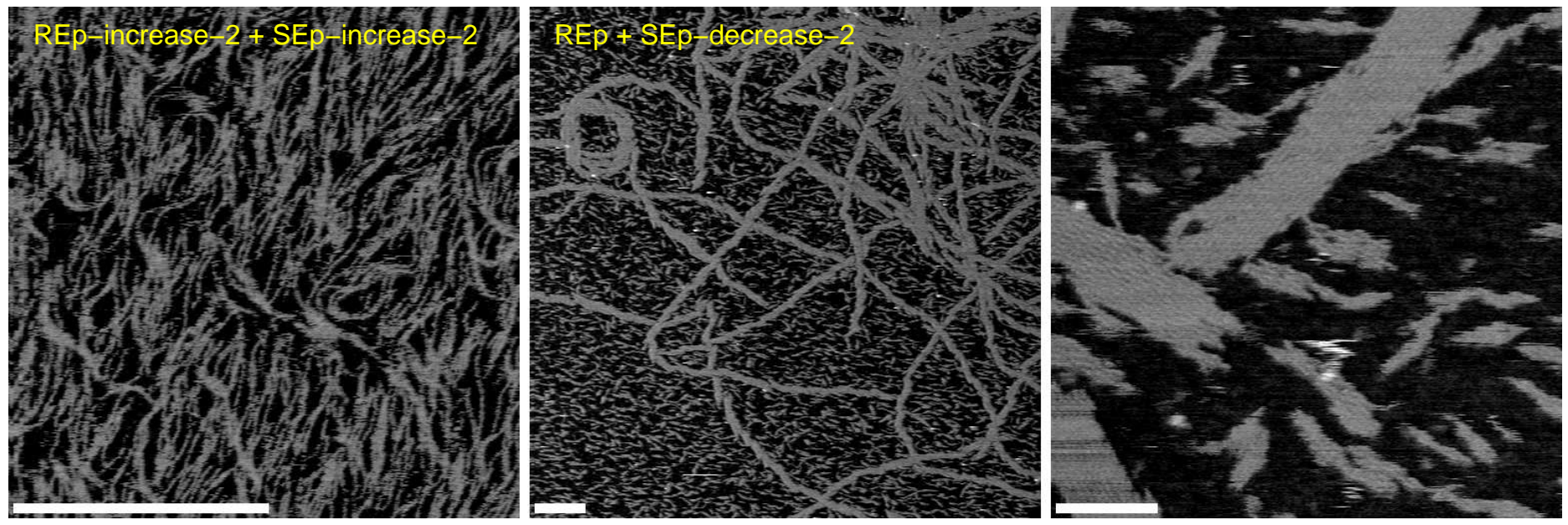

Figure 16. (left) Tangled fi laments produced by REp + SEp each with arm lengths increased by 2. Scale bar $500 \mathrm{~nm}$. (middle) Normal REp combined with SEp-decrease-2 with hairpin at position 13. Tubes appear to rip diagonally, producing a distinct feathered appearance. (right) Zoom suggests that tubes are helical, with exactly one tile column offset. Scale bar, $100 \mathrm{~nm}$. 


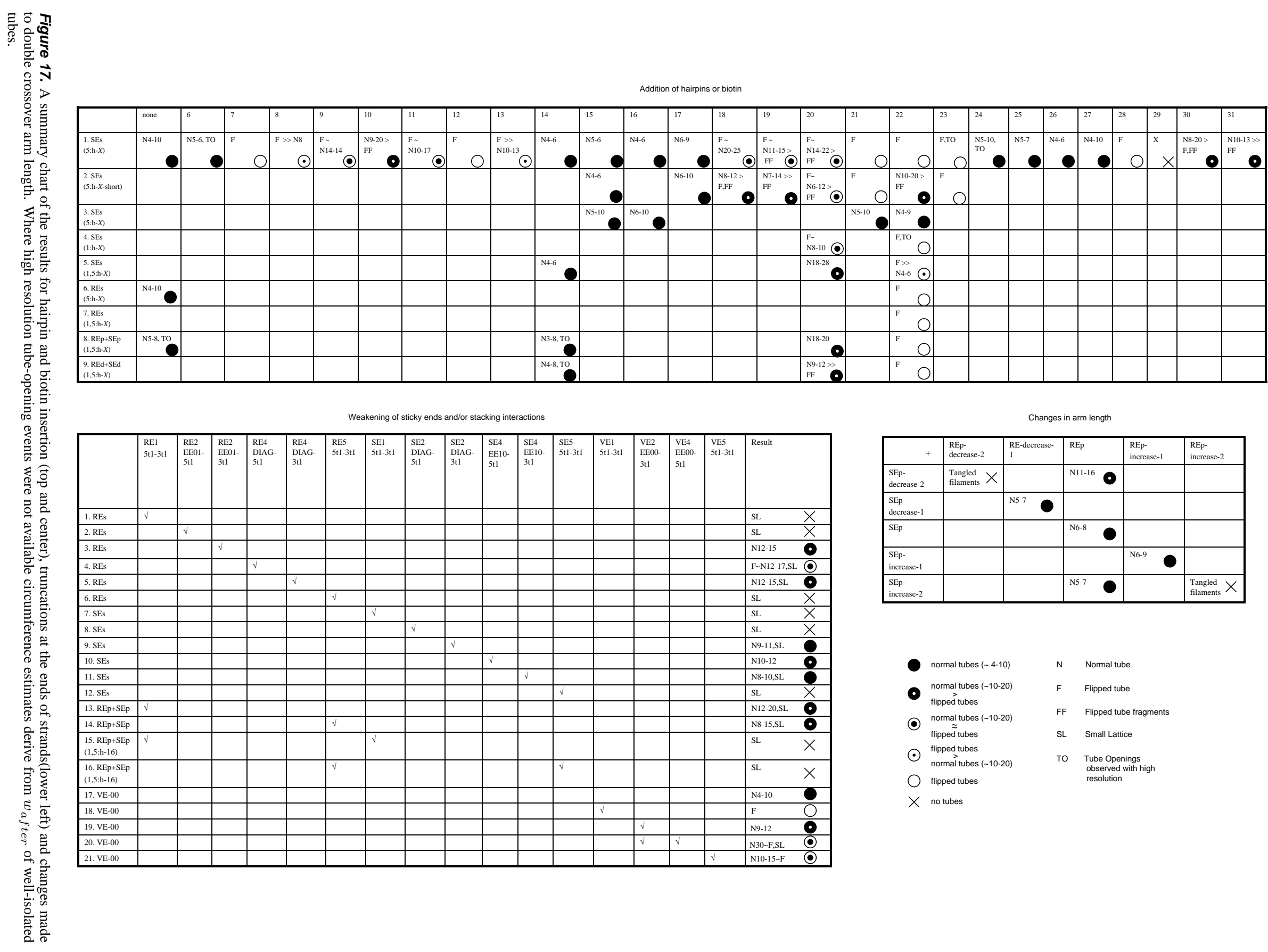

